\title{
Bioactive Phytochemicals with Anti-Aging and Lifespan Extending Potentials in Caenorhabditis elegans
}

\author{
Nkwachukwu Oziamara Okoro ${ }^{1,2,3,+}$, Arome Solomon Odiba ${ }^{1,2,+}$, Patience Ogoamaka Osadebe ${ }^{3}$, \\ Edwin Ogechukwu Omeje ${ }^{3}$, Guiyan Liao ${ }^{4}$, Wenxia Fang ${ }^{2,4}$ (D), Cheng Jin 1,2,5 (D) and Bin Wang 1,2,*(D) \\ 1 National Engineering Research Center for Non-Food Biorefinery, Guangxi Academy of Sciences, \\ Nanning 530007, China; nkwachukwu.okoro.pg01008@unn.edu.ng (N.O.O.); \\ arome.odiba@unn.edu.ng (A.S.O.); jinc@im.ac.cn (C.J.) \\ 2 College of Life Science and Technology, Guangxi University, Nanning 530007, China; wfang@gxas.cn \\ 3 Department of Pharmaceutical and Medicinal Chemistry, University of Nigeria, Nsukka 410001, Nigeria; \\ patience.osadebe@unn.edu.ng (P.O.O.); Edwin.omeje@unn.edu.ng (E.O.O.) \\ 4 State Key Laboratory of Non-Food Biomass and Enzyme Technology, Guangxi Academy of Sciences, \\ Nanning 530007, China; gyliao@gxas.cn \\ 5 Institute of Microbiology, Chinese Academy of Sciences, Beijing 100101, China \\ * Correspondence: bwang@gxas.cn; Tel.: +86-771-2503-601 \\ + Okoro Nkwachukwu Oziamara and Arome Solomon Odiba contributed equally to this work.
}

check for

updates

Citation: Okoro, N.O.; Odiba, A.S.; Osadebe, P.O.; Omeje, E.O.; Liao, G.; Fang, W.; Jin, C.; Wang, B. Bioactive Phytochemicals with Anti-Aging and Lifespan Extending Potentials in Caenorhabditis elegans. Molecules 2021, 26, 7323. https://doi.org/10.3390/ molecules 26237323

Academic Editors: Severina Pacifico and H. P. Vasantha Rupasinghe

Received: 3 November 2021

Accepted: 29 November 2021

Published: 2 December 2021

Publisher's Note: MDPI stays neutral with regard to jurisdictional claims in published maps and institutional affiliations.

Copyright: (c) 2021 by the authors. Licensee MDPI, Basel, Switzerland. This article is an open access article distributed under the terms and conditions of the Creative Commons Attribution (CC BY) license (https:/ / creativecommons.org/licenses/by/ $4.0 /)$.

\begin{abstract}
In the forms of either herbs or functional foods, plants and their products have attracted medicinal, culinary, and nutraceutical applications due to their abundance in bioactive phytochemicals. Human beings and other animals have employed those bioactive phytochemicals to improve health quality based on their broad potentials as antioxidant, anti-microbial, anti-carcinogenic, antiinflammatory, neuroprotective, and anti-aging effects, amongst others. For the past decade and half, efforts to discover bioactive phytochemicals both in pure and crude forms have been intensified using the Caenorhabditis elegans aging model, in which various metabolic pathways in humans are highly conserved. In this review, we summarized the aging and longevity pathways that are common to C. elegans and humans and collated some of the bioactive phytochemicals with health benefits and lifespan extending effects that have been studied in C. elegans. This simple animal model is not only a perfect system for discovering bioactive compounds but is also a research shortcut for elucidating the amelioration mechanisms of aging risk factors and associated diseases.
\end{abstract}

Keywords: aging; bioactive compounds; Caenorhabditis elegans; longevity pathways; lifespan

\section{Introduction}

Various plants and their by-products have become a major area of investigation for bioactive compounds with health benefits [1,2]. These bioactive phytochemicals are chemical components (mostly secondary metabolites) that are present in relatively smaller amounts compared to macronutrients such as carbohydrates, proteins, and lipids. Depending on the specific application and benefits, these phytochemicals are classified into various categories such as medicinal, functional foods, nutraceuticals, and botanicals, some of which are closely related or almost convey the same meaning. These compounds function in other processes that are vital to plant survival, such as protection and adaptation, due to their inability to escape several potential categories of ecological and environmental (biotic and abiotic) damaging stresses. However, their benefits extend to humans and other animals that have taken advantage of these properties by sourcing such valuable components from plants for other benefits beyond the basic macronutrients that are essential for life. Most of the bioactive substances that have been discovered from food sources so far are mostly of plant origin and are consumed as fruits, legumes, vegetables, spices, and medicinal herbs [2,3]. These products are available in diverse forms, such as in fresh, raw, or processed forms. Beyond their nutritional values, these food sources 
contain bioactive compounds that exert anti-microbial, anti-viral, anti-carcinogenic, antiinflammatory, antioxidants, neuroprotective, and anti-aging effects [2]. Recent research in this field has sought to discover new natural bioactive compounds in food forms that have anti-aging and lifespan extending benefits, examples of which include Silymarin and 6-shogaol [1,3-7].

Aging in humans is closely associated with diverse pathological changes, including cancer, cardiovascular disorders, metabolic diseases such as type II diabetes, and neurodegenerative diseases such as Alzheimer's disease [1,2]. Conserved in all living forms, aging is a degenerative process that is characterized by a progressive deterioration of cellular components and functions, which in most cases, inevitably leads to mortality [3,4]. In developed countries, aging accounts for $90 \%$ of deaths, with about 100,000 cases per day, which makes up approximately two-thirds of deaths globally [5]. The United Nations projects that by 2050, the proportion of the global populace older that is older than 60 years of age will be closely doubled from 962 million to 2.1 billion [6]. Therefore, it is critical to gain an understanding of the molecular mechanism of the aging process along with the search for therapeutic interventions that are capable of extending lifespan and improving health span.

There are nine widely acknowledged hallmarks of aging that feature all of the major alterations of the key biological functions: loss of proteostasis, genomic instability, telomere attrition, mitochondrial dysfunction, epigenetic alterations, cellular senescence, deregulated nutrient-sensing, stem cell exhaustion, and altered intercellular communication [7]. In particular, the proteostasis network, which consists of protein synthesis, folding, secretion, trafficking, disaggregation, and degradation [7,8], is an integral part of the biological quality control systems that ensure cellular homeostasis for the survival and propagation of the organism. During aging, the decline of the proteostasis network contributes to the development of proteotoxicity related disorders such as Parkinson's disease, Huntington's disease, Alzheimer's disease, and Amyotrophic lateral sclerosis [1,9]. There are two prominent concepts or theories as to how adverse changes occur in aging at the molecular level [10]. The programmed theory proposes aging as a genetically programmed chronic process, whereas the damage theory puts emphasis on the gradual and cumulative damage to cells and organs derived from internal and external factors [11]. The damage theory largely stems from the free radical theory that primarily focuses on the generation of reactive oxygen species (ROS) as metabolic by-products and their accumulative damage to biomolecules [10-13] such as DNA/RNA, proteins, and lipids. The antidotes to this fundamental challenge are the antioxidants that provide a counter mechanism to keep the balance of ROS production in check. On one hand, it secures all of the essential ROS-dependent reactions, and on the other, it removes excessive ROS and prevents the undesirable species from being generated, thereby delaying aging-associated changes and increasing longevity $[4,11,12,14]$. Meanwhile, the search for bioactive compounds (from natural sources as well as synthetic) possessing antioxidant properties has been greatly intensified, which has so far brought to light several candidates with interesting potential in enhancing lifespan and health span.

Many critical aspects of the human aging process have been studied in model organisms, providing us great insight into the individual elements and more importantly the operating and underlying regulatory mechanisms. Amongst these organisms, the Caenorhabditis elegans model has been greatly employed for the discovery of longevity pathways as well as new anti-aging compounds with lifespan extending properties [15-18]. Here, we briefly introduce C. elegans as an important aging model, highlighting the prominent pathways or factors that are involved in aging process, and discussed the recent discovery of lifespan-extending compounds, plant sources as well as their anti-aging activities in the C. elegans system. 


\section{C. elegans as a Model for Aging Research}

Model organisms have become a crucial part of biomedical research, tackling fundamental biological and medical questions that would otherwise be impossible to study in humans due to the cost, system complexity, and ethical issues [19-21]. Any biological system in which aging occurs has the potential of being a model for studying aging. However, the choice of the model is largely dependent on the specific questions to be answered as well as the amenability of the model. C. elegans, a multicellular organism sharing $60-80 \%$ similarity with humans at the genomic level, has emerged as an outstanding model for aging research $[22,23]$. Featured by a highly conserved aging signaling network, C. elegans has a relatively short life cycle and low maintenance and propagation costs [24,25]. More so, sophisticated genetic techniques and manipulations, such as RNAi, CRISPR-cas9, and the auxin-inducible degradation (AID) system, are all applicable to $C$. elegans for transgenesis as well as forward and reverse genetic screening [26]. The transparent body of $C$. elegans also serves an ideal system for real-time live imaging of fluorescence-tagged proteins in the whole animal [27-29]. Despite the abounding advantages with using C. elegans in aging research, it is not without limitations. On the downside, the advantages that are associated with the small size of this organism, such as ease of handling, can also be a substantial disadvantage, as obtaining a large amount of the same generation is limiting as well as labor intensive. Likewise, in spite of the $>60 \%$ genetic conservation with humans, C. elegans is indeed a lower invertebrate and is significantly distant from mammals evolutionarily, biochemically, and physiologically [30-32].

A series of pioneering works established the biochemical pathways that are associated with aging and longevity in C. elegans, which appear to be conserved through evolution [31-36]. Klass isolated eight mutant strains with a remarkable increase in lifespan and correlated this phenomenon with the restriction of caloric intake [33]. Further studies led to the identification of age-1, the first gene linked to lifespan extension [37,38]. Similarly, mutations in daf-2 exhibited more than a double-fold increase in the lifespan through regulating the activity of daf-16 [36]. These discoveries set up the foundation for using the C. elegans model to understand the aging process and to seek opportunities for lifespan extension. More work was inspired, collectively leading to the appreciation of the complex network underpinning the aging process. Meanwhile, this model has also been explored in other dimensions, ranging from assessing the environmental factors for aging (hermetic treatments and caloric restriction), studying the age-related diseases, population and evolutionary studies, and screening of drugs with potential lifespan-extending properties [13,39-42].

\section{Signaling Pathways and Environmental Factors Related to Aging}

Over 70 genes have been implicated in the pathways regulating the lifespan of $C$. elegans [38], with a high likelihood of further expansion on the current number. These genes are involved in the nutrient-sensing signaling pathways, including Target of Rapamycin (mTOR) signaling, AMP-activated protein kinase (AMPK)-dependent signaling, sirtuins, and insulin/IGF-1 signaling (IIS). Other implicated pathways include the JNK pathway, TGF- $\beta$ signaling, germline signaling, and mitochondrial respiration as well as other factors leading to aging process such as protein homeostasis, temperature, transcription factors, and so on. The representative genes that are involved in those pathways are listed in Figure 1.

Currently, daf-16 is the most central aging related gene and is the downstream target of some of the pathways, most prominently, the IIS pathway. Its activity involves direct interaction with other genes, modulating the nuclear translocation or acting as a transcription factor to numerous target genes for lifespan regulation and stress resistance [43-46]. Not surprisingly, the pathways or factors that are involved in the aging process interact with each other to mediate lifespan extension. 


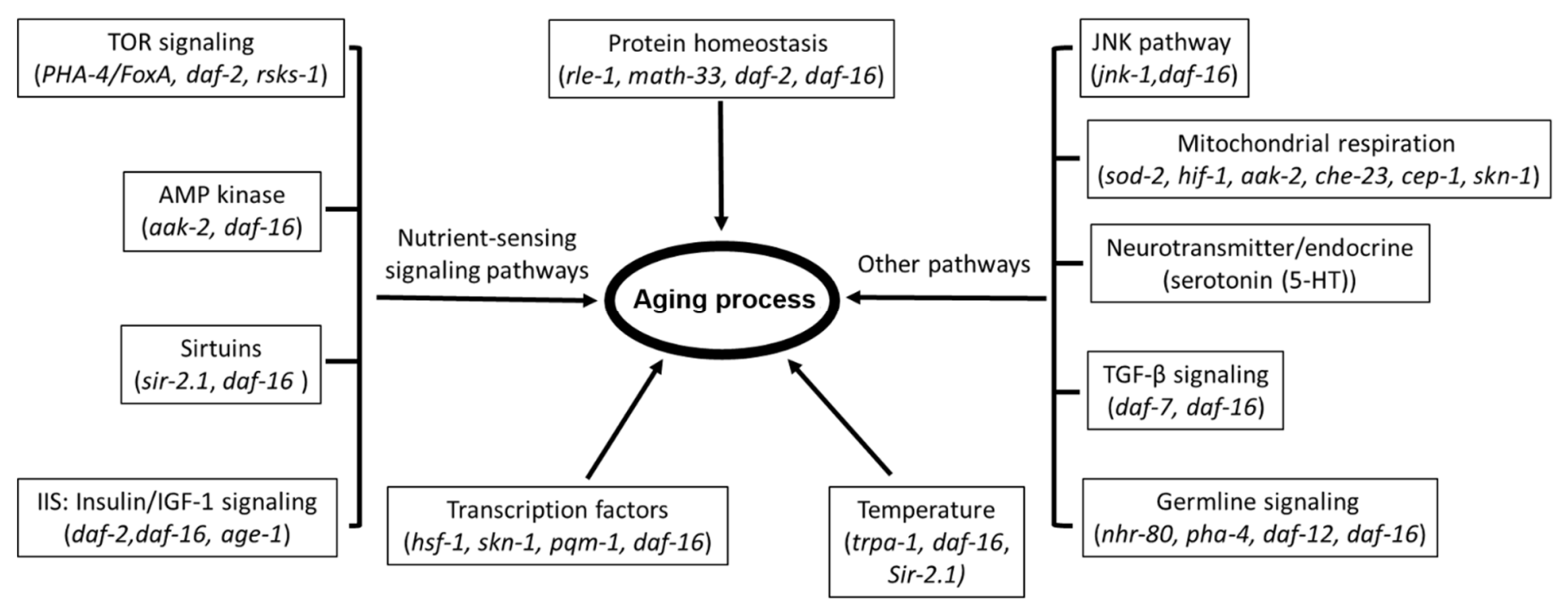

Figure 1. The known signaling pathways and key factors linked to aging process.

\section{Bioactive Phytochemicals with Health Benefits}

Derived from natural sources such as plants, animals, and microorganisms, bioactive compounds exhibit a highly diverse chemical nature. They are capable of interacting with biochemical systems, thereby conferring a wide spectrum of health benefits such as anti-inflammatory, anti-aging, anti-hypertensive, anti-cancer, anti-diabetic, and antineurodegenerative properties [47-53]. Of all of the bioactive compounds, the phytochemicals from plants are the most prominent and have gained more attention since increasing numbers of lifespan-extending candidates have been discovered from phenotypic screening in C. elegans [54]. Reverse genetic screening along with structural studies has elucidated the targets of some of these compounds and the related pathways that are involved. Phytochemical/secondary metabolites can be found in vegetables, fruits, cereals, and inedible plants, and many of them have been identified to possess antioxidant properties [55]. They are chemically categorized into polyphenols, terpenoids, alkaloids, saponins, phytosterols, and organosulfur compounds [55-59]. Here we focused on the application of the most abundant and most chemically diverse phytochemicals (polyphenols, terpenoids, and alkaloids) to aging studies and further highlighted the use of crude extract in aging studies in the subsequent section.

\subsection{Polyphenolic Compounds}

The polyphenolic compounds include flavonoids, tannins, stilbenes, coumarins, lignans, and other phenolic compounds (Table 1) that are mainly involved in curbing oxidative stress and related conditions by providing their reducing power to protect essential cellular components from detrimental oxidative damage [60]. Among the members of this class, the flavonoids are the most abundant group, with over 8000 compounds having been identified [61]. Flavonoids contain a basic flavan nucleus with 15 carbon atoms that are grouped into $\mathrm{C}_{6}-\mathrm{C}_{3}-\mathrm{C}_{6}$ skeleton that comprises two aromatic $\mathrm{C}_{6}$ rings and a heterocyclic ring with one oxygen atom [62]. The presence of a highly reactive hydroxyl group enables flavonoids to donate the hydrogen atom, thereby reducing highly oxidizing free radicals [62]. The main targeted pathway linked to the anti-aging efficacy by this class of compounds is IIS. For example, Tambulin, as a hydroxy substituted flavanol, enhances stress tolerance and longevity and mitigates the manifestation of Parkinson-like symptoms in C. elegans model, during which the IIS pathway is up-regulated by the expressions of daf-16, sod-1, sod-3, and ctl-2 [22]. Rosmarinic acid (RA) as a natural polyphenol, has been shown to improve the mean lifespan of $C$. elegans [63] by up-regulating the IIS pathway via ins-18 and daf-16; the MAPK pathway via skn-1 and sek-1; and the stress resistance and antioxidant 
genes such as $c t l-1$, sod-3 and sod-5. The lifespan of the worms can also be extended by curcumin [64], which depends on the functions of age-1, skn-1, sir-2.1, sek-1, unc-43, osr-1, and $m e k-1$, which are related to the IIS, MAPKK, and JNK signaling pathways. Polyphenol and chlorogenic acid enhance longevity via the IIS pathway depending on daf-16, skn-1 and $h s f-1$ [65]. Epigallocatechin gallate and epicatechin (EC) protect and facilitate the expression of major genes of the IIS pathway to protect $C$. elegans from oxidative stress, thereby enhancing the longevity [66-68]. Furthermore, pro-longevity effects have been found in the $C$. elegans aging models, with a broad variety of polyphenols/flavonoids such as myricetin, resveratrol, quercetin, naringenin, kaempferol, catechin, baicalein, fisetin, caffeic acid, phenethylester, acacetin, and blueberry polyphenols, most of which require daf-16 [42,69-80]. 
Table 1. Polyphenolic compounds with anti-aging and lifespan extending properties as demonstrated in the C. elegans model.

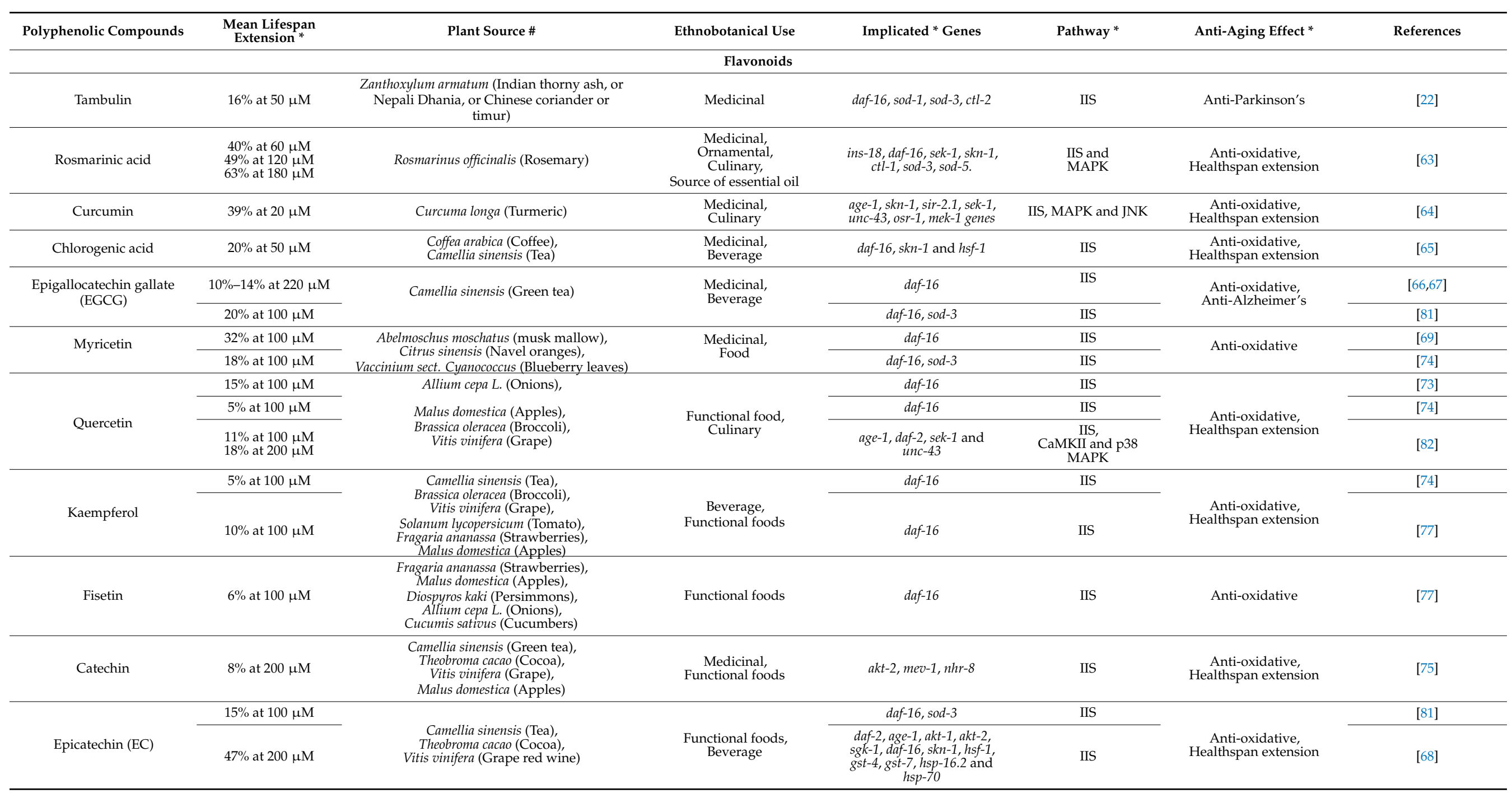


Table 1. Cont.

\begin{tabular}{|c|c|c|c|c|c|c|c|}
\hline Polyphenolic Compounds & $\begin{array}{c}\text { Mean Lifespan } \\
\text { Extension * }\end{array}$ & Plant Source \# & Ethnobotanical Use & Implicated ${ }^{*}$ Genes & Pathway * & Anti-Aging Effect * & References \\
\hline 3'-O-methylepicatechin & $6 \%$ at $200 \mu \mathrm{M}$ & $\begin{array}{l}\text { Malus domestica (Apples), } \\
\text { Vitis vinifera (Grape), }\end{array}$ & Functional foods, & - & - & Anti-oxidative, & [83] \\
\hline 4'-O-methylepicatechin & $12 \%$ at $200 \mu \mathrm{M}$ & $\begin{array}{l}\text { Theobroma cacao (Cocoa), } \\
\text { Camellia sinensis (Tea) }\end{array}$ & Beverage & - & - & Healthspan extension & [83] \\
\hline Baicalein & $36 \%$ at $0.1 \% w / v$ & $\begin{array}{l}\text { Scutellaria baicalensis (Baikal skullcap or } \\
\text { Chinese skullcap) }\end{array}$ & Medicinal & $c b p-1$ & - & Anti-oxidative & [84] \\
\hline Caffeic acid & $11 \%$ at $300 \mu \mathrm{M}$ & Coffea arabica (Coffee) & $\begin{array}{l}\text { Beverage, } \\
\text { Medicinal }\end{array}$ & $\begin{array}{l}o s r-1, \text { sek-1, sir-2.1, unc-43, } \\
\text { and daf }-16\end{array}$ & & Anti-oxidative, & [85] \\
\hline $\begin{array}{c}\text { Acacetin } \\
\text { (5,7-dihydroxy-4- } \\
\text { methoxyflavone) }\end{array}$ & $27 \%$ at $25 \mu \mathrm{M}$ & $\begin{array}{l}\text { Tephroseris kirilowii (Dog Tongue grass, Cotton } \\
\text { bat) }\end{array}$ & Medicinal & $\begin{array}{c}\text { sod-3, gst-4, ctl-1 and } \\
h s p-16.2\end{array}$ & IIS & $\begin{array}{l}\text { Anti-oxidative, } \\
\text { Stress Resistance }\end{array}$ & [86] \\
\hline $\begin{array}{c}\text { Acacetin 7-O- } \alpha-1- \\
\text { rhamnopyranosyl(1-2) } \beta- \\
\text { D-xylopyranoside }\end{array}$ & $39 \%$ at $25 \mu \mathrm{M}$ & Premna integrifolia (Wind killer) & Medicinal & $\begin{array}{c}\text { sir-2.1, skn-1, daf- } 16 \text {, and } \\
h s f-1\end{array}$ & & $\begin{array}{l}\text { Anti-oxidative, } \\
\text { Stress Resistance }\end{array}$ & [87] \\
\hline $\begin{array}{l}\text { Quercetin-3-O- } \\
\text { dirhamnoside } \\
\end{array}$ & $21 \%$ at $200 \mu \mathrm{M}$ & Curcuma longa (turmeric) & $\begin{array}{l}\text { Culinary, } \\
\text { Medicinal }\end{array}$ & - & - & $\begin{array}{c}\text { Anti-oxidative, } \\
\text { Stress Resistance }\end{array}$ & [39] \\
\hline Quercetin-3-O-glucoside & $23 \%$ at $25 \mu \mathrm{M}$ & Erica multiflora (Winter Heather) & Medicinal & - & - & Anti-oxidative & [88] \\
\hline $\begin{array}{l}\text { Isorhamnetin (Quercetin } \\
\text { 3'-O-methylether) }\end{array}$ & $16 \%$ at $200 \mu \mathrm{M}$ & $\begin{array}{c}\text { Ginkgo biloba (ginkgo or gingko also known as } \\
\text { the maidenhair tree), } \\
\text { Hippophae rhamnoides (Sea buckthorn), } \\
\text { Vaccinium sect. Cyanococcus (Blueberry) }\end{array}$ & $\begin{array}{l}\text { Medicinal, } \\
\text { Food }\end{array}$ & - & - & $\begin{array}{l}\text { Anti-oxidative, } \\
\text { Stress Resistance }\end{array}$ & [89] \\
\hline Icariin & $20 \%$ at $45 \mu \mathrm{M}$ & Herba epimedii (Horny Goat Weed) & Medicinal & daf-2, daf- $16, h s f-1$ & IIS & $\begin{array}{c}\text { Anti-oxidative, } \\
\text { Anti-neurodegenerative } \\
\text { diseases }\end{array}$ & [90] \\
\hline Icariside II & $20 \%$ at $20 \mu \mathrm{M}$ & Herba epimedii (Horny Goat Weed) & Medicinal & daf-2, daf- $16, h s f-1$ & IIS & $\begin{array}{c}\text { Anti-oxidative, } \\
\text { Stress resistance, } \\
\text { Anti-neurodegenerative } \\
\text { diseases }\end{array}$ & [90] \\
\hline Isoxanthohumol & $2 \%$ at $100 \mu \mathrm{M}$ & Humulus lupulus (hops) & $\begin{array}{l}\text { Medicinal } \\
\text { Beverage }\end{array}$ & daf-16 & IIS & $\begin{array}{l}\text { Anti-oxidative, } \\
\text { Stress resistance }\end{array}$ & [91] \\
\hline Silymarin & $\begin{array}{l}10 \% \text { at } 25 \mu \mathrm{M} \\
24 \% \text { at } 50 \mu \mathrm{M}\end{array}$ & Silybum marianum (Milk thistle) & Medicinal & - & - & $\begin{array}{l}\text { Anti-oxidative, } \\
\text { Stress resistance, } \\
\text { Anti-Alzheimer's }\end{array}$ & [1] \\
\hline Genistein & $27 \%$ at $100 \mu \mathrm{M}$ & Vigna angularis (adzuki bean) & $\begin{array}{l}\text { Medicinal, } \\
\text { Food }\end{array}$ & hsp 16.2, sod-3 & & $\begin{array}{l}\text { Healthspan extension, } \\
\text { Stress resistance }\end{array}$ & [92] \\
\hline Taxifolin & $51 \%$ at $820 \mu \mathrm{M}$ & $\begin{array}{l}\text { Silybum marianum (blessed thistle or milk } \\
\text { thistle), } \\
\text { Carduus marianus (Marian thistle or } \\
\text { Our-Lady's-thistle), } \\
\text { Allium cepa L. (Onions) }\end{array}$ & $\begin{array}{l}\text { Medicinal, } \\
\text { Culinary }\end{array}$ & - & - & $\begin{array}{l}\text { Stress Resistance, } \\
\text { Ameliorates Cerebral } \\
\text { Amyloid Angiopathy } \\
\text { (ACAA) }\end{array}$ & [93] \\
\hline
\end{tabular}


Table 1. Cont.

\begin{tabular}{|c|c|c|c|c|c|c|c|}
\hline Polyphenolic Compounds & $\begin{array}{c}\text { Mean Lifespan } \\
\text { Extension }\end{array}$ & Plant Source \# & Ethnobotanical Use & Implicated $*$ Genes & Pathway * & Anti-Aging Effect * & References \\
\hline $\begin{array}{c}\text { Trolox } \\
\text { (6-Hydroxy-2,5,7,8- } \\
\text { tetramethylchroman-2- } \\
\text { carboxylic } \\
\text { acid }\end{array}$ & $\begin{array}{c}31 \% \text { at } 0.6 \mathrm{mM}-3 \\
\mathrm{mM}\end{array}$ & Fragaria ananassa (Strawberries) & Functional food & - & - & Anti-oxidative & [93] \\
\hline Chicoric Acid & $20 \%$ at $100 \mu \mathrm{M}$ & $\begin{array}{l}\text { Cichorium intybus (Chicory), } \\
\text { Echinacea angustifolia (Purple cone flower), } \\
\text { Lactuca sativa (Lettuce), } \\
\text { Ocimum basilicum (Basil) }\end{array}$ & $\begin{array}{l}\text { Medicinal, } \\
\text { Culinary }\end{array}$ & - & AMPK & Anti-oxidative & [94] \\
\hline Naringin & $23 \%$ at $50 \mu \mathrm{M}$ & $\begin{array}{c}\text { Citrus grandis (Pomelo), Citrus paradise } \\
\text { (grapefruit), and Citrus aurantium (Bitter } \\
\text { orange) }\end{array}$ & Functional food & $\begin{array}{l}\text { daf- } 16, \text { daf-2, akt-1, akt-2. } \\
\text { eat-2, sir-2.1, rsks-1, } \\
\text { and clk-1 }\end{array}$ & IIS & $\begin{array}{l}\text { Anti-oxidative, } \\
\text { Anti-Alzheimer's, } \\
\text { Anti-Parkinson's }\end{array}$ & [35] \\
\hline \multicolumn{8}{|c|}{ Tannins } \\
\hline & $18 \%$ at $100 \mu \mathrm{M}$ & & \multirow[b]{2}{*}{ Functional food } & sek-1 & MAPK & \multirow{2}{*}{$\begin{array}{c}\text { Anti-oxidative, } \\
\text { Anti-Alzheimers', } \\
\text { Neuroprotective } \\
\text { Others } \\
\text { Anti-amyloidogenic, } \\
\text { Antimicrobial, Anticancer, } \\
\text { Antimutagenic }\end{array}$} & [95] \\
\hline Tannic acid & $24 \%$ at $0.01 \% w / v$ & $\begin{array}{l}\text { Camellia sinensis (Tea), } \\
\text { Vitis vinifera (Grape), } \\
\text { Arachis hypogaea (Pea nuts) }\end{array}$ & & daf-16 & IIS & & [84] \\
\hline Pentagalloyl Glucose & $18 \%$ at $160 \mu \mathrm{M}$ & $\begin{array}{l}\text { Eucalyptus leaves (Southern blue gum or blue } \\
\text { gum) }\end{array}$ & & $\begin{array}{l}\text { daf- } 16, \text { age-1, eat-2, sir-2.1 } \\
\text { and isp-1 }\end{array}$ & $\begin{array}{l}\text { IIS, DR, SIR-2.1 and } \\
\text { METC. }\end{array}$ & $\begin{array}{c}\text { Anti-oxidative } \\
\text { Others } \\
\text { Estrogenic, } \\
\text { Anti-inflammatory, } \\
\text { Anti-oxidative, Anticancer }\end{array}$ & [53] \\
\hline \multicolumn{8}{|c|}{ Stilbene } \\
\hline \multirow{3}{*}{ Resveratrol } & $\begin{array}{c}\text { Variable effects at } \\
100 \mu \mathrm{M}\end{array}$ & \multirow{3}{*}{$\begin{array}{c}\text { Vitis vinifera (Grape), } \\
\text { Peanuts, } \\
\text { Theobroma cacao (Cocoa), } \\
\text { Vaccinium sect. Cyanococcus (Blueberry), } \\
\text { Vaccinium myrtillus (Bilberry), } \\
\text { Vaccinium macrocarpon (cranberry) }\end{array}$} & \multirow{3}{*}{ Functional food } & sir 2.1 & - & \multirow{3}{*}{$\begin{array}{c}\text { Anti-oxidative } \\
\text { Others } \\
\text { Antiviral, } \\
\text { Anti-depressant, } \\
\text { Anti-nociceptive, } \\
\text { Anti-diabetic activities }\end{array}$} & [70] \\
\hline & $3 \%$ at $5 \mu \mathrm{M}$ & & & - & - & & [71] \\
\hline & $11 \%$ at $100 \mu \mathrm{g} / \mathrm{mL}$ & & & & - & & [72] \\
\hline OxyResveratrol & $31 \%$ at $1000 \mu \mathrm{M}$ & Morus alba (white mulberry) & $\begin{array}{l}\text { Functional food, } \\
\text { Medicine }\end{array}$ & sir-2.1, aak & AMPK and SIR-2.1 & $\begin{array}{l}\text { Anti-oxidative, } \\
\text { Neuroprotective }\end{array}$ & [96] \\
\hline $\begin{array}{c}\text { TSG }\left(2,3,5,4^{\prime}-\right. \\
\text { Tetrahydroxystilbene-2-O- } \\
\beta \text {-D-glucoside) }\end{array}$ & $23 \%$ at $100 \mu \mathrm{M}$ & Polygonum multiflorum (Tuber fleeceflower) & Medicinal & - & - & $\begin{array}{l}\text { Anti-oxidative, } \\
\text { Stress resistance }\end{array}$ & [97] \\
\hline Polydatin & $30 \%$ at $1 \mathrm{mM}$ & Vitis vinifera (Grape) & Functional food & $\begin{array}{c}\text { sir-2.1, skn-1, sod-3, and } \\
\text { daf- } 16\end{array}$ & IIS & $\begin{array}{l}\text { Anti-oxidative, } \\
\text { Stress resistance, } \\
\text { Neuroprotective }\end{array}$ & [98] \\
\hline Piceatannol & $\underset{\mu \mathrm{M}}{18 \% \text { at } 50 \text { and } 100}$ & $\begin{array}{l}\text { Passiflora edulis (Passion fruit), } \\
\text { Camellia sinensis (White Tea), } \\
\text { Vitis vinifera (Grape) }\end{array}$ & Functional food & $\underset{\text { dir-2.1 }}{\operatorname{daf}-16, \operatorname{hsp}^{\prime} 16.2, \text { sod-3, }}$ & IIS & $\begin{array}{l}\text { Anti-oxidative } \\
\text { Others } \\
\text { Estrogenic, } \\
\text { Anti-inflammatory, } \\
\text { Anti-oxidative, } \\
\text { Anticancer }\end{array}$ & [99] \\
\hline
\end{tabular}


Table 1. Cont.

\begin{tabular}{|c|c|c|c|c|c|c|c|}
\hline Polyphenolic Compounds & $\begin{array}{c}\text { Mean Lifespan } \\
\text { Extension* }\end{array}$ & Plant Source \# & Ethnobotanical Use & Implicated ${ }^{*}$ Genes & Pathway * & Anti-Aging Effect * & References \\
\hline \multicolumn{8}{|c|}{ Coumarins } \\
\hline ^Urolithin A (UA) & $45 \%$ at $50 \mu \mathrm{M}$ & $\begin{array}{c}\text { Vaccinium sect. Cyanococcus (Blueberry), } \\
\text { Fragaria ananassa (Strawberries), Arachis } \\
\text { hypogaea (Pea nuts), Quercus spp (acorns), } \\
\text { Punica granatum (pomegranates), Juglans regia } \\
\text { (Walnut), Rubus idaeus (raspberries) }\end{array}$ & Functional food & - & - & $\begin{array}{l}\text { Anti-oxidative, } \\
\text { Healthspan extension }\end{array}$ & [100] \\
\hline${ }^{\wedge}$ Urolithin B (UB) & $36 \%$ at $50 \mu \mathrm{M}$ & $\begin{array}{l}\text { Vaccinium sect. Cyanococcus (Blueberry), } \\
\text { Fragaria ananassa (Strawberries), Arachis } \\
\text { hypogaea (Pea nuts), Quercus spp (acorns), } \\
\text { Punica granatum (pomegranates), Juglans regia } \\
\text { (Walnut), Rubus idaeus (raspberries) }\end{array}$ & Functional food & - & - & $\begin{array}{l}\text { Anti-oxidative, } \\
\text { Healthspan extension }\end{array}$ & [100] \\
\hline `Urolithin C (UC) & $36 \%$ at $50 \mu \mathrm{M}$ & $\begin{array}{l}\text { Vaccinium sect. Cyanococcus (Blueberry), } \\
\text { Fragaria ananassa (Strawberries), Arachis } \\
\text { hypogaea (Pea nuts), Quercus spp (acorns), } \\
\text { Punica granatum (pomegranates), Juglans regia } \\
\text { (Walnut), Rubus idaeus (raspberries) }\end{array}$ & Functional food & - & - & $\begin{array}{l}\text { Anti-oxidative, } \\
\text { Healthspan extension }\end{array}$ & [100] \\
\hline ^urolithin D (UD) & $19 \%$ at $50 \mu \mathrm{M}$ & $\begin{array}{c}\text { Vaccinium sect. Cyanococcus (Blueberry), } \\
\text { Fragaria ananassa (Strawberries), Arachis } \\
\text { hypogaea (Pea nuts), Quercus spp (acorns), } \\
\text { Punica granatum (pomegranates), Juglans regia } \\
\text { (Walnut), Rubus idaeus (raspberries) }\end{array}$ & Functional food & - & - & $\begin{array}{l}\text { Anti-oxidative, } \\
\text { Healthspan extension }\end{array}$ & [100] \\
\hline \multicolumn{8}{|c|}{ Lignan } \\
\hline Vitexin & $17 \%$ at $100 \mathrm{mM}$ & Vigna angularis (adzuki beans) & Functional Food & sod-3, hsp-16.2 & IIS & $\begin{array}{l}\text { Anti-oxidative } \\
\text { Others } \\
\text { Antiviral, } \\
\text { Anti-depressant, } \\
\text { Anti-nociceptive, } \\
\text { Anti-diabetic }\end{array}$ & [13] \\
\hline Arctigenin & $13 \%$ at $100 \mu \mathrm{M}$ & Arctium lappa (Greater burdock) & Medicinal & daf-16, jnk-1 & IIS & $\begin{array}{l}\text { Anti-oxidative, } \\
\text { Stress resistance }\end{array}$ & [102] \\
\hline Matairesinol & $25 \%$ at $100 \mu \mathrm{M}$ & Arctium lappa (Greater burdock) & Medicinal & daf-16, jnk-1 & IIS & $\begin{array}{l}\text { Anti-oxidative, } \\
\text { Stress resistance }\end{array}$ & [102] \\
\hline Arctiin & $15 \%$ at $100 \mu \mathrm{M}$ & Arctium lappa (Greater burdock) & Medicinal & daf-16, jnk-1 & IIS & $\begin{array}{l}\text { Anti-oxidative, } \\
\text { Stress resistance }\end{array}$ & [102] \\
\hline
\end{tabular}


Table 1. Cont.

\begin{tabular}{|c|c|c|c|c|c|c|c|}
\hline Polyphenolic Compounds & $\begin{array}{c}\text { Mean Lifespan } \\
\text { Extension * }\end{array}$ & Plant Source \# & Ethnobotanical Use & Implicated ${ }^{*}$ Genes & Pathway * & Anti-Aging Effect * & References \\
\hline Lappaol C & $11 \%$ at $100 \mu \mathrm{M}$ & Arctium lappa (Greater burdock) & Medicinal & daf-16, jnk-1 & IIS & $\begin{array}{l}\text { Anti-oxidative, } \\
\text { Stress resistance }\end{array}$ & [102] \\
\hline Lappaol F & $12 \%$ at $100 \mu \mathrm{M}$ & Arctium lappa (Greater burdock) & Medicinal & daf-16, jnk-1 & IIS & $\begin{array}{l}\text { Anti-oxidative, } \\
\text { Stress resistance }\end{array}$ & [102] \\
\hline (Iso) lappaol A & $11 \%$ at $100 \mu \mathrm{M}$ & Arctium lappa (Greater burdock) & Medicinal & daf-16, jnk-1 & IIS & $\begin{array}{l}\text { Anti-oxidative, } \\
\text { Stress resistance }\end{array}$ & [102] \\
\hline \multicolumn{8}{|c|}{ Phenolic Compounds } \\
\hline Tryosol & $21 \%$ at $250 \mu \mathrm{M}$ & Olea Europea L. (Olive tree) & $\begin{array}{l}\text { Medicinal, } \\
\text { Food }\end{array}$ & $h s f-1$, daf- 2, daf -16 & IIS & $\begin{array}{l}\text { Anti-oxidative, } \\
\text { Stress resistance }\end{array}$ & [103] \\
\hline 6-Shogaol & $\begin{array}{c}19 \% \text { at } 12.5 \mu \mathrm{M} \\
25 \% \text { at } 25 \mu \mathrm{M}\end{array}$ & Zingiber officinale (Ginger) & $\begin{array}{l}\text { Culinary, } \\
\text { Medicinal }\end{array}$ & sod-3, hsp-16.2 & & $\begin{array}{l}\text { Anti-oxidative, } \\
\text { Stress resistance }\end{array}$ & [5] \\
\hline Salicyclic Acid & $14 \%$ at $1 \mathrm{mM}$ & $\begin{array}{l}\text { Rubus idaeus (raspberries), } \\
\text { Salix alba L. (Willow tree) }\end{array}$ & $\begin{array}{l}\text { Medicinal, } \\
\text { Food }\end{array}$ & $\begin{array}{c}\text { daf-16, sod-3, sod-5, ctl-2, } \\
\text { gst-4, sst-10 }\end{array}$ & IIS & $\begin{array}{l}\text { Anti-oxidative, } \\
\text { Stress resistance }\end{array}$ & [104] \\
\hline Salicylamine & $\begin{array}{l}32 \% \text { at } 100 \mu \mathrm{M} \\
56 \% \text { at } 500 \mu \mathrm{M}\end{array}$ & Fagopyrum esculentum (Buckwheat) & Culinary & sir-2.1, ets-7 & - & Healthspan extension & [105] \\
\hline Juglone & $29 \%$ at $40 \mu \mathrm{M}$ & Juglans nigra (Black Walnut) & $\begin{array}{l}\text { Functional Food, } \\
\text { Medicinal }\end{array}$ & $\begin{array}{c}\text { daf-16, sod-3, hsp-16.2, } \\
\text { sir-2.1 }\end{array}$ & IIS & $\begin{array}{l}\text { Anti-oxidative, } \\
\text { Stress resistance }\end{array}$ & [106] \\
\hline Gallic Acid & $25 \%$ at $300 \mu \mathrm{M}$ & $\begin{array}{l}\text { Punica granatum (Pomegranate), } \\
\text { Aspalathus linearis (Rooibos tea), } \\
\text { Vitis vinifera (Grape), } \\
\text { Raphanus sativus (Black radish), } \\
\text { Allium cepa L. (Onions) }\end{array}$ & Functional food & - & - & Stress resistance & [17] \\
\hline Ferulic Acid & $9.58 \%$ at $500 \mu \mathrm{M}$ & $\begin{array}{l}\text { Beta vulgaris (Beet root), Oryza sativa (Rice), } \\
\text { Glycine max (Soyabean), Daucus carota } \\
\text { (Carrot), Avena sativa (Oats) }\end{array}$ & Functional food & $\begin{array}{l}\text { daf-2, daf }-16, \text { hlh-30, skn-1, } \\
\text { and hsf-1 }\end{array}$ & IIS & $\begin{array}{c}\text { Healthspan extension, } \\
\text { Stress resistance, } \\
\text { Anti- Huntington's disease }\end{array}$ & [107] \\
\hline
\end{tabular}

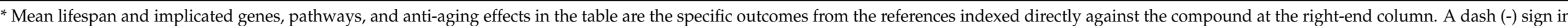

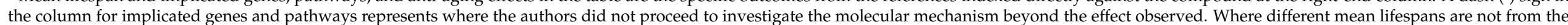

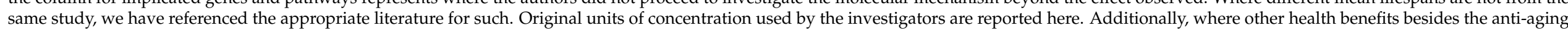

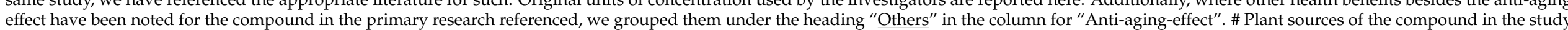

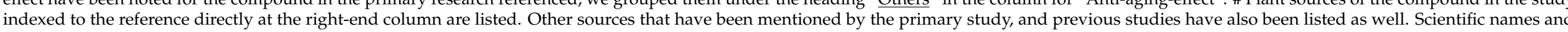

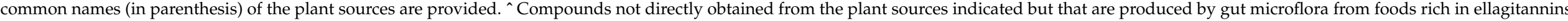
such as the plants listed. 


\subsection{Terpenoids}

Terpenoids are the most abundant and structurally diverse phytochemicals. Also known as terpenes, they can be classified based on the number of isoprene units $\left(\mathrm{C}_{5} \mathrm{H}_{8}\right)_{\mathrm{n}}$ into various subgroups, including hemiterpenes $\left(\mathrm{C}_{5} \mathrm{H}_{8}\right)$, monoterpenes $\left(\mathrm{C}_{10} \mathrm{H}_{16}\right)$, sesquiterpenes $\left(\mathrm{C}_{15} \mathrm{H}_{24}\right)$, diterpenes $\left(\mathrm{C}_{20} \mathrm{H}_{32}\right)$, sesterterpenes $\left(\mathrm{C}_{25} \mathrm{H}_{40}\right)$, triterpenes $\left(\mathrm{C}_{30} \mathrm{H}_{48}\right)$, tetraterpenes $\left(\mathrm{C}_{40} \mathrm{H}_{64}\right)$, and polyterpenes $\left(\mathrm{C}_{5} \mathrm{H}_{8}\right)_{\mathrm{n}}$. Terpenoids exhibit a broad range of pharmacological activities, ranging from anti-malarial, anti-cancer, anti-inflammatory, anti-bacterial, anti-viral, and anti-aging to anti-neurodegeneration [108]. Some of the notable terpenoids are the chemotherapy medication $\mathrm{Taxol}^{\circledR}$ (paclitaxel) and the frontline antimalarial drug Artemisinin [108].

Isoprenol is a hemiterpene-based unsaturated $C_{5}$ alcohol that confers lifespan extension and stress tolerance in C. elegans via daf-16 and skn-1 through the IIS pathway [23]. Moreover, the expressions of SOD-3 and GST- 4 can be boosted by isoprenol through the translocation of DAF-16 from the cytosol into the nucleus [22]. Carnosic acid relies on the MAPK and HSF-1 pathway to up-regulate sod-5, hsp-16.2, hsp-16.1, sek-1, and skn-1 [109]. Carnosol, as a phenolic diterpene, increases the mean lifespan of $C$. elegans through the HSF-1 signaling pathway to up-regulate sod-3 and sod-5 as antioxidants and $h s p-16.1$ and hsp-16.2 for heat shock response [110]. Beta-caryophyllene (BCP), a naturally occurring bicyclic sesquiterpene, is capable of extending lifespan as well as of increasing the resistance to oxidative stress by inducing the dietary restriction response and xenobiotic stress response [111]. The compound 4-Hydroxy-E-globularinin, an iridoid isolated from Premma integrifolia, exhibits detoxification activity against ROS and up-regulates $h s p-16.2$ and sod-3 through daf-16 in the IIS pathway [112]. A similar effect was found with oleanolic acid, where sod-3, hsp-16.2, and ctl-1 were up-regulated via daf-16 [113]. However, $\alpha$-Tocopherol, either in free form or encapsulated with SDNF (soluble dietary fiber-based nanofibers), may provide the longevity benefits via different routes [114]. Lifespan extension potentials have also been ascribed to additional high molecular weight isoprenoid-based compounds such as withanolide-A, specioside, ursolic acid, and glycyrrhetinic acid [60,115-117]. Examples of these terpenoids with longevity-modulating effects are summarized in Table 2.

Table 2. Terpenoids with anti-aging and lifespan extending properties as demonstrated in the C. elegans model.

\begin{tabular}{|c|c|c|c|c|c|c|c|}
\hline Terpenoids & $\begin{array}{c}\text { Mean } \\
\text { Lifespan } \\
\text { Extension * }\end{array}$ & Plant Source \# & $\begin{array}{c}\text { Ethnobotanical } \\
\text { Use }\end{array}$ & $\begin{array}{l}\text { Implicated * } \\
\text { Genes }\end{array}$ & Pathway * & $\underset{\text { Effect }}{\text { Anti-Aging }}$ & References \\
\hline Carnosic Acid & $\begin{array}{c}3 \% \text { at } 60 \mu \mathrm{M} \\
8 \% \text { at } 120 \mu \mathrm{M} \\
16 \% \text { at } 180 \mu \mathrm{M}\end{array}$ & $\begin{array}{l}\text { Rosmarinus } \\
\text { officinalis } L \\
\text { (Rosemary) }\end{array}$ & $\begin{array}{c}\text { Food, } \\
\text { Medicinal }\end{array}$ & $\begin{array}{c}\text { sod-5, hsp-16.2, } \\
\text { hsp-16.1, sek-1, } \\
\text { skn-1 }\end{array}$ & $\begin{array}{l}\text { MAPK and } \\
\text { HSF-1 }\end{array}$ & $\begin{array}{c}\text { Anti-oxidant, } \\
\text { Anti- } \\
\text { inflammatory, } \\
\text { Antibacterial, } \\
\text { Anti-cancer, } \\
\text { Neuroprotec- } \\
\text { tive }\end{array}$ & [109] \\
\hline Carnosol & $19 \%$ at $180 \mu \mathrm{M}$ & $\begin{array}{l}\text { Rosmarinus } \\
\text { officinalis } L \\
\text { (Rosemary) }\end{array}$ & $\begin{array}{l}\text { Medicinal, } \\
\text { Culinary }\end{array}$ & $\begin{array}{c}\text { sod-3, sod-5, } \\
\text { hsp-16.1, } \\
\text { hsp-16.2, hsf-1, } \\
\text { daf-16. }\end{array}$ & IIS & $\begin{array}{c}\text { Antioxidant, } \\
\text { Anticancer, } \\
\text { Antimicrobial, } \\
\text { Anti- } \\
\text { inflammatory }\end{array}$ & [110] \\
\hline $\begin{array}{c}\text { Beta- } \\
\text { Caryophyllene }\end{array}$ & $>22 \%$ at $50 \mu \mathrm{M}$ & $\begin{array}{c}\text { Syzygium } \\
\text { aromaticum (Clove), } \\
\text { Cannabis sativa } \\
\text { (hemp), } \\
\text { Rosmarinus } \\
\text { officinalis } L \\
\text { (Rosemary, } \\
\text { Humulus lupulus } \\
\text { (Hops) }\end{array}$ & $\begin{array}{l}\text { Culinary, } \\
\text { Beverages }\end{array}$ & $\begin{array}{c}\text { pha-4, sir-2.1, } \\
\text { hsf-1, skn-1, } \\
\text { daf-16, gst-4, } \\
\text { gst-7, hsp-70, } \\
\text { sod-2, sod-3 and } \\
\text { daf-9 }\end{array}$ & IIS & $\begin{array}{l}\text { Anti-oxidant, } \\
\text { Anti- } \\
\text { inflammatory, } \\
\text { anti-biotic, } \\
\text { Anti- } \\
\text { carcinogenic, } \\
\text { local anesthetic }\end{array}$ & {$[111]$} \\
\hline
\end{tabular}


Table 2. Cont.

\begin{tabular}{|c|c|c|c|c|c|c|c|}
\hline Terpenoids & $\begin{array}{c}\text { Mean } \\
\text { Lifespan } \\
\text { Extension * }\end{array}$ & Plant Source \# & $\begin{array}{c}\text { Ethnobotanical } \\
\text { Use }\end{array}$ & $\begin{array}{l}\text { Implicated * } \\
\text { Genes }\end{array}$ & Pathway * & $\underset{\text { Effect* }}{\text { Anti-Aging }}$ & References \\
\hline $\begin{array}{l}\text { 4-Hydroxy-E- } \\
\text { globularinin }\end{array}$ & $18 \%$ at $20 \mu \mathrm{M}$ & $\begin{array}{l}\text { Premna integrifolia } \\
\text { (Wind killer) }\end{array}$ & Medicinal & $\begin{array}{l}\text { daf-16, hsp-16.2, } \\
\text { sod-3 }\end{array}$ & IIS & Anti-oxidant & [112] \\
\hline $\begin{array}{l}\text { 10-O-trans- } p \text { - } \\
\text { Coumaroylcatalpol }\end{array}$ & $17 \%$ at $20 \mu \mathrm{M}$ & $\begin{array}{l}\text { Premna integrifolia } \\
\text { (Wind killer) }\end{array}$ & Medicinal & daf-16 & IIS & $\begin{array}{l}\text { Anti-oxidant, } \\
\text { Anti- } \\
\text { parkinson's } \\
\text { disease }\end{array}$ & [118] \\
\hline Oleanolic acid & $16 \%$ at $300 \mu \mathrm{M}$ & $\begin{array}{l}\text { Constituent of the } \\
\text { leaves } \\
\text { and roots of more } \\
\text { than } 120 \text { plant } \\
\text { species such as } \\
\text { Olea europaea (olive } \\
\text { tree), } \\
\text { Viscum album } \\
\text { (European } \\
\text { mistletoe or } \\
\text { common mistletoe), } \\
\text { Aralia chinensis } \\
\text { (Chinese Angelica } \\
\text { Tree) }\end{array}$ & $\begin{array}{l}\text { Food, } \\
\text { Medicinal }\end{array}$ & $\begin{array}{l}\text { sod-3, hsp-16.2, } \\
\text { ctl-1, daf-16 }\end{array}$ & IIS & $\begin{array}{l}\text { Anti-oxidant, } \\
\text { Hepatoprotective, } \\
\text { Hypoglycemic, } \\
\text { Anti- } \\
\text { inflammatory }\end{array}$ & [113] \\
\hline$\alpha$-Tocopherol & $\begin{array}{c}7 \% \text { at } 50 \\
\mu \mathrm{g} / \mathrm{mL} \\
15 \% \text { at } 100 \\
\mu \mathrm{g} / \mathrm{mL} \\
17 \% \text { at } 200 \\
\mu \mathrm{g} / \mathrm{mL}\end{array}$ & $\begin{array}{l}\text { Sunflower seeds } \\
\text { (Helianthus } \\
\text { annuus), } \\
\text { Prunus dulcis } \\
\text { (Almonds), } \\
\text { Corylus avellana L. } \\
\text { (Hazelnuts), } \\
\text { Arachis hypogaea } \\
\text { (Pea nuts), } \\
\text { Spinacia oleracea } \\
\text { (Spinach), } \\
\text { Brassica oleracea var. } \\
\text { italica (Broccoli), } \\
\text { Actinidia deliciosa } \\
\text { (Kiwifruit), } \\
\text { Mangifera indica } \\
\text { (Mango) }\end{array}$ & $\begin{array}{l}\text { Functional } \\
\text { food }\end{array}$ & - & - & Anti-oxidant & [114] \\
\hline Withanolide-A & $29 \%$ at $5 \mu \mathrm{M}$ & $\begin{array}{l}\text { Withania Somnifera } \\
\text { (Ashwagandha) }\end{array}$ & Medicinal & $\begin{array}{l}\text { sgk-1, daf-16, } \\
\text { sod-3, skn-1, } \\
\text { hsf- } 1, \text { gst-4, } \\
\text { hsp-16.2 }\end{array}$ & IIS & $\begin{array}{l}\text { Neuroprotective, } \\
\text { Stress } \\
\text { resistance }\end{array}$ & [115] \\
\hline Specioside & $15 \%$ at $25 \mu \mathrm{M}$ & $\begin{array}{c}\text { Stereospermum } \\
\text { suaveolens (Patala) }\end{array}$ & Medicinal & $\begin{array}{c}\text { sod-1, sod-2, } \\
\text { sod-3, gst-4, } \\
\text { gst-7, hsp-16.2, } \\
\text { hsp-70, clt-1 }\end{array}$ & IIS & $\begin{array}{l}\text { Antioxidant, } \\
\text { Stress } \\
\text { resistance }\end{array}$ & [87] \\
\hline Ursolic acid & $32 \%$ at $25 \mu \mathrm{M}$ & $\begin{array}{l}\text { Malus domestica } \\
\text { (Apple peels), } \\
\text {, rosemary, } \\
\text { Lavandula } \\
\text { angustifolia } \\
\text { (lavender), Mentha } \\
\text { piperita } \\
\text { (Peppermint), } \\
\text { Thymus vulgaris } \\
\text { (thyme), Ocimum } \\
\text { basilicum (Basil), } \\
\text { Vaccinium myrtillus } \\
\text { (Bilberry) }\end{array}$ & $\begin{array}{l}\text { Medicinal, } \\
\text { Culinary }\end{array}$ & $j n k-1, j k k-1$ & JNK-1 & $\begin{array}{l}\text { Antioxidant, } \\
\text { Stress } \\
\text { resistance }\end{array}$ & [117] \\
\hline $\begin{array}{l}18 \alpha- \\
\text { Glycyrrhetinic } \\
\text { acid }\end{array}$ & $\begin{array}{l}17 \% \text { at } 20 \\
\mu \mathrm{g} / \mathrm{mL}\end{array}$ & $\begin{array}{l}\text { Glycyrrhiza glabra } \\
\text { (Licorice) }\end{array}$ & $\begin{array}{l}\text { Medicinal, } \\
\text { Culinary }\end{array}$ & skn-1, daf-16 & p38 MAPK & Neuroprotective & [59] \\
\hline Glaucarubinone & $\begin{array}{c}1.9 \text { days at } 100 \\
\mathrm{nM}\end{array}$ & $\begin{array}{l}\text { Simaroubaceae spp. } \\
\text { (Amargo, } \\
\text { Bitterwood, } \\
\text { Marupa, or } \\
\text { Quassia) }\end{array}$ & $\begin{array}{l}\text { Ornamental, } \\
\text { Medicinal }\end{array}$ & - & - & $\begin{array}{l}\text { Anti-oxidant } \\
\text { Others } \\
\text { Antimalarial }\end{array}$ & [71] \\
\hline
\end{tabular}


Table 2. Cont.

\begin{tabular}{|c|c|c|c|c|c|c|c|}
\hline Terpenoids & $\begin{array}{c}\text { Mean } \\
\text { Lifespan } \\
\text { Extension * }\end{array}$ & Plant Source \# & $\begin{array}{l}\text { Ethnobotanical } \\
\text { Use }\end{array}$ & $\begin{array}{l}\text { Implicated * } \\
\text { Genes }\end{array}$ & Pathway * & $\underset{\text { Effect }}{\text { Anti-Aging }}$ & References \\
\hline Fucoxanthin & $14 \%$ at $5 \mu \mathrm{M}$ & $\begin{array}{c}\text { Undaria pinnatifida } \\
\text { (Wakame), } \\
\text { Hijikia fusiformis } \\
\text { (Hijiki) }\end{array}$ & Medicinal & - & - & $\begin{array}{l}\text { Antioxidant, } \\
\text { Stress } \\
\text { resistance }\end{array}$ & [119] \\
\hline Catalpol & $28 \%$ at $25 \mu \mathrm{M}$ & $\begin{array}{l}\text { Rehmannia glutinosa } \\
\text { (Chinese foxglove) }\end{array}$ & $\begin{array}{l}\text { Medicinal } \\
\text { plant }\end{array}$ & $\begin{array}{l}\text { mek-1, daf-2, } \\
\text { age-1, daf-16, } \\
\text { and skn-1 }\end{array}$ & IIS & $\begin{array}{l}\text { Anti-oxidant, } \\
\text { Anti- } \\
\text { Alzheimer's, } \\
\text { Anti- } \\
\text { Parkinson's, } \\
\text { Anti-stroke } \\
\text { Others } \\
\text { Anticancer, } \\
\text { Anti-diabetes }\end{array}$ & [120] \\
\hline Ferulsinaic acid & $20 \%$ at $100 \mu \mathrm{M}$ & $\begin{array}{l}\text { Ferula communis } \\
\text { (Giant Fennel) }\end{array}$ & $\begin{array}{l}\text { Medicinal, } \\
\text { Culinary }\end{array}$ & - & - & Anti-oxidant & [121] \\
\hline Verminoside & $20 \%$ at $25 \mu \mathrm{M}$ & $\begin{array}{c}\text { Stereospermum } \\
\text { suaveolens (Patala) }\end{array}$ & Medicinal & daf-16 & - & $\begin{array}{l}\text { Antioxidant, } \\
\text { Stress } \\
\text { resistance }\end{array}$ & [116] \\
\hline $\begin{array}{l}\text { Dehydroabietic } \\
\text { acid }\end{array}$ & $15 \%$ at $10 \mu \mathrm{M}$ & $\begin{array}{c}\text { Pinus densiflora } \\
\text { (Japanese red pine), } \\
\text { Pinus sylvestris } \\
\text { (Scots pine), } \\
\text { Abies } \\
\text { grandis(Grand fir) }\end{array}$ & Medicinal & sir-2.1 & - & $\begin{array}{l}\text { Healthspan } \\
\text { extension }\end{array}$ & [122] \\
\hline $\begin{array}{c}\text { Secoisolariciresinol } \\
\text { Diglucoside }\end{array}$ & $22 \%$ at $500 \mu \mathrm{M}$ & $\begin{array}{l}\text { Linum } \\
\text { usitatissimum } \\
\quad(\text { Flaxseed })\end{array}$ & $\begin{array}{l}\text { Food, } \\
\text { Medicine }\end{array}$ & $\begin{array}{c}\text { daf- } 16, \text { hsf-1, } \\
\text { nhr-80, daf-12, } \\
\text { glp-1, eat-2, and } \\
\text { aak-2. }\end{array}$ & IIS & $\begin{array}{l}\text { Anti-oxidant, } \\
\text { Anti- } \\
\text { Alzheimer's, } \\
\text { Anti- } \\
\text { Parkinson's }\end{array}$ & [123] \\
\hline
\end{tabular}

* Mean lifespan and implicated genes, pathways, and anti-aging effects in the table are the specific outcomes from the references indexed directly against the compound at the right-end column. A dash (-) sign in the column for implicated genes and pathways represents where authors did not proceed to investigate the molecular mechanism beyond the effect observed. Where different mean lifespans are not from the same study, we have referenced the appropriate literature for such. Original units of concentration used by the investigators are reported here. Additionally, where other health benefits besides the anti-aging effect have been noted for the compound in the primary research referenced, we grouped them under the heading "Others" in the column for "Anti-aging-effect". \# Plant sources of the compound in the study indexed to the reference directly at the right-end column are listed. Other sources that have been mentioned by the primary study and previous studies have also been listed as well. Scientific names and common names (in parenthesis) of the plant sources are provided.

\subsection{Alkaloids}

Alkaloids represent a class of nitrogenous chemicals that are not only derived from plants but also from fungi, bacteria, and animals [124]. According to their heterocyclic ring system and biosynthetic precursor, this class of compounds are categorized into eight subgroups, including tropanes, indoles, imidazoles, piperidines, isoquinolines, pyrrolizidines, quinolozidines, and pyrrolidine alkaloids [124]. Historically, some alkaloids have been used as poisons, whereas others have been used as remedies against fever and snakebites [124-126]. Though a bit under the shadow of toxicity, alkaloids elicit great potential in pharmaceutical development, primarily as analgesic, antioxidant, anti-inflammatory, anti-bacterial, anti-spasmodic, anti-cancer, anti-hypertensive, and stimulants to the central nervous system [56,124,127]. Particularly, over 300 compounds of this class have been shown to possess some degree of anti-aging property [40]. Reserpine confers significant thermo tolerance and longevity benefits to the worms, which is likely independent of daf-16 and partially rely on serotonin [126]. Enhanced longevity effects are also offered by the methylxanthine alkaloid caffeine, which is able to induce the nuclear translocation of daf-16 but does not requires its activity [128]. The action of pentagalloyl glucose (a gallotannin) on lifespan requires the cooperation between four pathways, including the IIS pathway, the mitochondrial ETC, the Sir-2.1 signaling, and the dietary restriction pathway [53]. The studied alkaloids with lifespan extending potentials in C. elegans are summarized in Table 3. 
Table 3. Alkaloids with anti-aging and lifespan extending properties as demonstrated in the C. elegans model.

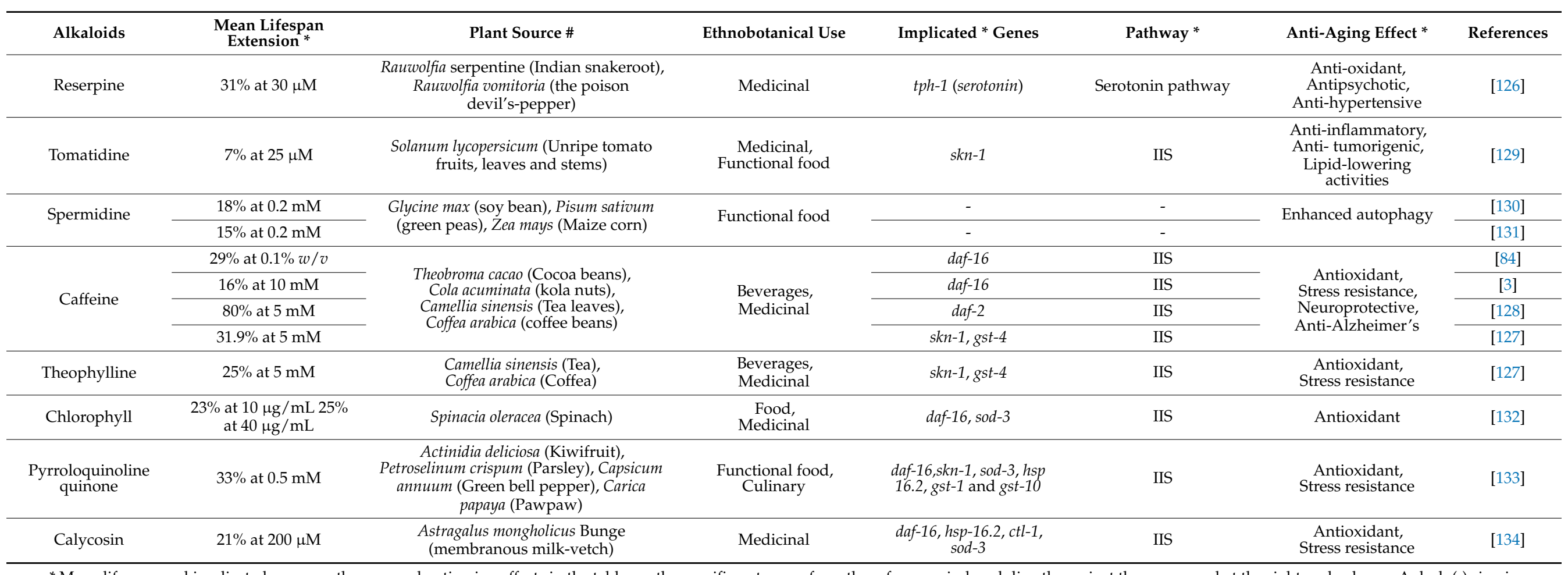

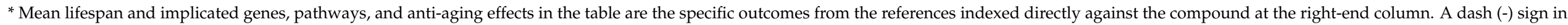

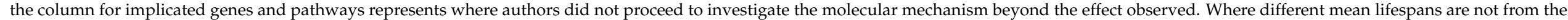

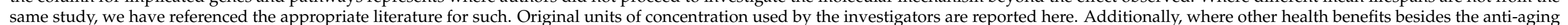

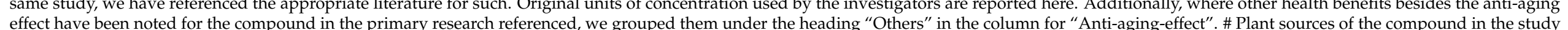

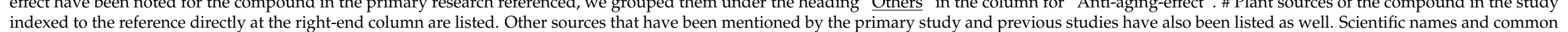
indexed to the reference directly at the right-end column a
names (in parenthesis) of the plant sources are provided. 


\subsection{Plants Crude Drugs and Extracts with Lifespan Extending Abilities in C. elegans}

Medicinal plants are essential sources of bioactive therapeutic compounds. Therapeutic studies using pure isolated phytochemicals are commonly preceded by preliminary studies using crude plant extracts to confirm the therapeutic potentials. Several studies have reported that some plant extracts show a lifespan prolonging effect on C. elegans. For instance, the aqueous stem bark extract from Endopleura uchi was reported to alter the DAF-16/FOXO pathway and to enhance the expression of the stress response genes such as hsp-16.2 and sod-3 [135]. Through High Performance Liquid Chromatography/UltravioletVisible (HPLC UV/VIS) analysis, phenolic bergenin has been proposed as the major active component, potentially paving a new path for further developing compounds with similar and even more potent effects [135]. Calycophyllum spruceanum water extract has been shown to modulate the DAF-16/FOXO pathway, and five secondary metabolites have been identified via HPLC/Mass Spectrometry (MS) analyses, including 5-hydroxy-6methoxycoumarin-7-glucoside, cyanidin, gardenoside, taxifolin, and 5-hydroxymorin [136]. Both the leaf and fruit extract of Caesalpinia mimosoides and Eugenia uniflora possess longevity enhancing activity via the IIS pathway with sod-3, gst-4, and $h s p-16.2$ as targets $[137,138]$. Glochidion zeylanicum and Anacardium occidentale leaf extracts have also been reported to display longevity enhancing and oxidative stress resistance activities in C. elegans via the DAF-16/FOXO and SKN-1/Nrf-2 signaling pathways [139,140]. Furthermore, phytochemical analysis narrowed down on the active compounds, revealing benzoic acid, pentadecanoic acid, octadecatrienoic acid, $n$-hexadecanoic acid, $\beta$-caryophyllene, palmitic acid, and $\alpha$-linolenic acid as prominent metabolites. Extract from Hibiscus sabdariffa $L$. exhibits significant lifespan extension activities alongside curbing amyloid- $\beta$ toxicity in C. elegans, which is mediated by the IIS pathway through the activation of the DAF-16 and SKN-1 transcription factors [141]. An ayurvedic polyherbal extract (PHE) derived from six herbs, including Berberis aristata, Emblica officinalis, Cyperus rotundus, Terminalia chebula Cedrus deodara, and Terminalia bellirica, has been shown to enhance the expressions of daf-16, daf-2, skn-1, sod-3, and gst-4, all of which are associated with longevity and stress response [142]. As mentioned in the foregoing, although crude extracts may exert therapeutic effects, the main ingredients that are responsible are pure chemical components that serve specific effects. It is therefore important to elucidate and characterize the specific components, as this will eventually pave the way for new strategic pharmacological designs. Examples of these plant extracts with longevity-modulating effects are summarized in Table 4.

Table 4. Plant crude extracts with anti-aging and lifespan-extending properties as demonstrated in the C. elegans model.

\begin{tabular}{|c|c|c|c|c|c|c|}
\hline Plant Source \# & $\begin{array}{c}\text { Mean Lifespan } \\
\text { Extension * }\end{array}$ & $\begin{array}{c}\text { Ethnobotanical } \\
\text { Use }\end{array}$ & $\underset{\text { Genes * }}{\text { Implicated }}$ & Pathway * & Anti-Aging Effect * & References \\
\hline $\begin{array}{c}\text { Endopleura uchi } \\
(U x i)\end{array}$ & $\begin{array}{c}33 \% \text { at } 300 \\
\mu \mathrm{g} / \mathrm{mL}\end{array}$ & Medicinal & $\begin{array}{l}\text { daf-16, hsp-16.2 } \\
\text { and sod-3 }\end{array}$ & IIS & $\begin{array}{c}\text { Antioxidant, } \\
\text { Stress resistance, } \\
\text { Anti-Huntington's } \\
\text { disease }\end{array}$ & {$[135]$} \\
\hline $\begin{array}{l}\text { Calycophyllum } \\
\text { spruceanum } \\
\text { (capirona) }\end{array}$ & $\begin{array}{c}16 \% \text { at } 300 \\
\mu \mathrm{g} / \mathrm{mL}\end{array}$ & Medicinal & daf-16 & IIS & $\begin{array}{l}\text { Antioxidant, } \\
\text { Stress resistance, } \\
\text { Healthspan } \\
\text { extension }\end{array}$ & [136] \\
\hline $\begin{array}{c}\text { Caesalpinia } \\
\text { mimosoides (Pansi) }\end{array}$ & $4 \%$ at $50 \mu \mathrm{g} / \mathrm{mL}$ & Food vegetable & daf -16, sod-3, gst-4 & IIS & $\begin{array}{c}\text { Antioxidant, } \\
\text { Stress resistance }\end{array}$ & {$[137]$} \\
\hline $\begin{array}{l}\text { Eugenia uniflora } \\
\text { (Surinam cherry) }\end{array}$ & $\begin{array}{c}\text { Significant } \\
\text { increase at } 500 \\
\mu \mathrm{g} / \mathrm{mL}\end{array}$ & $\begin{array}{c}\text { Food, } \\
\text { Medicinal }\end{array}$ & $\begin{array}{l}\text { daf-16, hsp-16.2 } \\
\text { and sod-3 }\end{array}$ & IIS & $\begin{array}{l}\text { Antioxidant, } \\
\text { Stress resistance, } \\
\text { Healthspan } \\
\text { extension }\end{array}$ & {$[138]$} \\
\hline
\end{tabular}


Table 4. Cont.

\begin{tabular}{|c|c|c|c|c|c|c|}
\hline Plant Source \# & $\begin{array}{c}\text { Mean Lifespan } \\
\text { Extension * }\end{array}$ & $\begin{array}{c}\text { Ethnobotanical } \\
\text { Use }\end{array}$ & $\begin{array}{c}\text { Implicated } \\
\text { Genes * }\end{array}$ & Pathway * & Anti-Aging Effect * & References \\
\hline $\begin{array}{c}\text { Anacardium } \\
\text { occidentale (Cashew) }\end{array}$ & $20 \%$ by $50 \mu \mathrm{g} / \mathrm{mL}$ & $\begin{array}{l}\text { Medicinal, } \\
\text { Functional food }\end{array}$ & $\begin{array}{l}\text { daf-16, skn-1, } \\
\text { sod-3, gst-4 }\end{array}$ & IIS & $\begin{array}{l}\text { Antioxidant, } \\
\text { Stress Resistance, } \\
\text { Healthspan } \\
\text { extension }\end{array}$ & [139] \\
\hline $\begin{array}{c}\text { Glochidion } \\
\text { zeylanicum } \\
\text { (Umbrella Cheese) }\end{array}$ & $\begin{array}{l}10 \% \text { at } 100 \\
\mu \mathrm{g} / \mathrm{mL}\end{array}$ & $\begin{array}{l}\text { Medicinal, } \\
\text { Food }\end{array}$ & $\begin{array}{l}\text { daf-16, skn-1, } \\
\text { sod-3, gst-4 }\end{array}$ & IIS & $\begin{array}{l}\text { Antioxidant, } \\
\text { Stress Resistance, } \\
\text { Healthspan } \\
\text { extension }\end{array}$ & [140] \\
\hline $\begin{array}{l}\text { Hibiscus sabdariffa L. } \\
\text { (Roselle) }\end{array}$ & $24 \%$ at $1 \mathrm{mg} / \mathrm{mL}$ & $\begin{array}{c}\text { Medicinal, } \\
\text { Beverage, } \\
\text { Food supplement }\end{array}$ & daf-16, skn-1 & IIS & $\begin{array}{c}\text { Antioxidant, } \\
\text { Stress Resistance, } \\
\text { Anti- } \\
\text { Neurodegenerative }\end{array}$ & [141] \\
\hline $\begin{array}{l}\text { Polyherbal extract of } \\
\text { Berberis aristata } \\
\text { (Indian barberry); } \\
\text { Emblica officinalis } \\
\text { (Indian gooseberry or } \\
\text { amla); Cyperus } \\
\text { rotundus (Purple } \\
\text { Nutsedge); } \\
\text { Terminalia chebula } \\
\text { (gall nut); Cedrus } \\
\text { deodara(Himalayan } \\
\text { cedar); Terminalia } \\
\text { bellirica (beleric } \\
\text { myrobalan) }\end{array}$ & $\begin{array}{c}16 \% \text { at } 0.01 \\
\mu \mathrm{g} / \mathrm{mL}\end{array}$ & Medicinal & $\begin{array}{l}\text { daf-16, daf-2, skn-1, } \\
\text { sod-3 and gst-4 }\end{array}$ & IIS & $\begin{array}{c}\text { Antioxidant, } \\
\text { Stress Resistance, } \\
\text { Anti- } \\
\text { Neurodegenerative }\end{array}$ & [142] \\
\hline $\begin{array}{c}\text { Betula utilis } \\
\text { (Himalayan Silver } \\
\text { Birch) }\end{array}$ & $\begin{array}{c}35.99 \% \text { at } 50 \\
\mu \mathrm{g} / \mathrm{mL}\end{array}$ & Medicinal & $\begin{array}{l}\text { daf-16, hsf-1, skn-1, } \\
\text { sod-3 and gst-4. }\end{array}$ & IIS & $\begin{array}{l}\text { Antioxidant, } \\
\text { Healthspan } \\
\text { extension }\end{array}$ & [143] \\
\hline $\begin{array}{l}\text { Citrus sinensis } \\
\text { (Orange extracts) }\end{array}$ & $\begin{array}{l}10.5 \%, 18.0 \% \text {, and } \\
26.2 \% \text { at } 100,200, \\
\text { and } 400 \mathrm{mg} / \mathrm{mL}, \\
\text { respectively }\end{array}$ & Functional food & $\begin{array}{l}\text { daf-16, sod-3, gst-4, } \\
\text { sek-1, and skn-1 }\end{array}$ & IIS & $\begin{array}{l}\text { Antioxidant, } \\
\text { Healthspan } \\
\text { extension }\end{array}$ & [35] \\
\hline $\begin{array}{l}\text { Cuscuta chinensis } \\
\text { (Chinese Dodder) }\end{array}$ & $24 \%$ at $30 \mu \mathrm{g} / \mathrm{mL}$ & Medicinal & $\begin{array}{c}h s p-16.1 \text { and } \\
h s p-12.6\end{array}$ & IIS & $\begin{array}{l}\text { Stress Resistance, } \\
\text { Healthspan } \\
\text { extension }\end{array}$ & {$[144]$} \\
\hline $\begin{array}{c}\text { Eucommia ulmoides } \\
\text { (Hardy Rubber } \\
\text { Tree) }\end{array}$ & $9 \%$ at $30 \mu \mathrm{g} / \mathrm{mL}$ & & - & - & Stress resistance & {$[144]$} \\
\hline
\end{tabular}

* Mean lifespan and implicated genes, pathways, and anti-aging effects in the table are the specific outcomes from the references indexed directly against the compound at the right-end column. A dash (-) sign in the column for implicated genes and pathways represents where authors did not proceed to investigate the molecular mechanism beyond the effect observed. Where different mean lifespans are not from the same study, we have referenced the appropriate literature for such. Original units of concentration used by the investigators are reported here. Additionally, where other health benefits besides the ant-aging effect have been noted for the compound in the primary research referenced, we grouped them under the heading "Others" in the column for "Anti-aging-effect". \# Plant sources of the compound in the study indexed to the reference directly at the right-end column are listed. Other sources that have been mentioned by the primary study and previous studies have also been listed along. Scientific names and common names (in parenthesis) of the plant sources are provided.

\section{Summary and Perspectives}

Globally, the significant rise in the aging populace is imposing a great economic and social burden. It is necessary to conduct more research focusing on the biological process of aging, with the aim of facilitating the development of potential interventions to alleviate the adverse health impact of aging-associated medical conditions such as neurodegenerative disorders, cancer, diabetes, and cardiovascular diseases. Suitable model organisms such as C. elegans will continuously provide more unique insight into this complex process as well as in the discovery of new bioactive compounds with pharmaceutical efficacy. Plant polyphenols including flavonoids, tannins, stilbene, coumarins, lignan, and other phenolic compounds as well as terpenoids and alkaloids have been a major source of these bioactive compounds, either as single purified compound or in crude extract mixtures. The disadvantage of crude extracts is that a complex interaction between all of the components of the crude and the biological system of the model may produce inhibitory, antagonistic, or synergistic effects. This makes it impossible to know exactly which component is inducing the particular effect being observed. Despite this challenge, crude the extracts of 
medicinal plants such as Endopleura uchi, Calycophyllum spruceanum, Caesalpinia mimosoides, Eugenia uniflora, Glochidion zeylanicum, Anacardium occidentale, Hibiscus sabdariffa L, and an ayurvedic polyherbal extract (PHE) derived from six herbs has demonstrated efficacy in extending lifespan in C. elegans [135-138,140-142]. However, purified compounds are better alternatives, as they provide the advantages of elucidating the structure, targets, mechanism of action, and pathways involved as well as the modification of the compound for improved activity.

In the future, discovering novel and effective natural candidates that extend lifespan and that delay aging and related diseases still depends on advancements in state-of-the-art high-throughput screening techniques $[145,146]$. Combining this effort with studies in suitable model organisms such as C. elegans $[147,148]$ will provide a better platform for understanding the aging process as well as facilitating healthy aging to improve the quality of life of the elderly population.

Author Contributions: N.O.O. and A.S.O. wrote the initial manuscript. P.O.O., E.O.O., G.L., C.J., W.F. and B.W. revised the manuscript. B.W. supervised the manuscript. All authors have read and agreed to the published version of the manuscript.

Funding: This research was funded by Research Start-up Funding of Guangxi Academy of Sciences (2017YJJ026), Collaborative Innovation Project of the Agricultural Science and Technology Innovation Program (CAAS-XTCX20190025-6), and Science and Technology Service Network Program of Chinese Academy of Sciences (KFJ-STS-QYZD-201-5-3) to B.W. and the Bagui Scholar Program Fund (2016A24) of Guangxi Zhuang Autonomous Region to C.J.

Conflicts of Interest: The authors declare no conflict of interest.

\section{References}

1. Kumar, J.; Park, K.-C.; Awasthi, A.; Prasad, B. Silymarin Extends Lifespan and Reduces Proteotoxicity in C. elegans Alzheimer's Model. CNS Neurol. Disord.-Drug Targets 2015, 14, 295-302. [CrossRef] [PubMed]

2. Sahardi, N.F.N.M.; Makpol, S. Ginger (Zingiber officinale Roscoe) in the Prevention of Ageing and Degenerative Diseases: Review of Current Evidence. Evid.-Based Complement. Altern. Med. 2019, 2019, 5054395. [CrossRef] [PubMed]

3. Sutphin, G.L.; Bishop, E.; Yanos, M.E.; Moller, R.M.; Kaeberlein, M. Caffeine extends life span, improves healthspan, and delays age-associated pathology in Caenorhabditis elegans. Longev. Healthspan 2012, 1, 9. [CrossRef] [PubMed]

4. Sadowska-Bartosz, I.; Bartosz, G. Effect of antioxidants supplementation on aging and longevity. Biomed Res. Int. 2014, 2014, 404680. [CrossRef]

5. Lee, E.B.; Kim, J.H.; Kim, Y.J.; Noh, Y.J.; Kim, S.J.; Hwang, I.H.; Kim, D.K. Lifespan-extending property of 6-shogaol from Zingiber officinale Roscoe in Caenorhabditis elegans. Arch. Pharm. Res. 2018, 41, 743-752. [CrossRef]

6. United Nations Department of Economic and Social Affairs: World Population Prospects; United Nations Department of Economic and Social Affairs: New York, NY, USA, 2017; pp. 1-25.

7. López-otín, C.; Blasco, M.A.; Partridge, L.; Serrano, M.; Kroemer, G. The Hallmarks of Aging Longevity. Cell 2013, $153,1194-1217$. [CrossRef] [PubMed]

8. Salminen, A.; Kaarniranta, K. AMP-activated protein kinase (AMPK) controls the aging process via an integrated signaling network. Ageing Res. Rev. 2012, 11, 230-241. [CrossRef]

9. Li, J.; Huang, K.X.; Le, W.D. Establishing a novel C. elegans model to investigate the role of autophagy in amyotrophic lateral sclerosis. Acta Pharmacol. Sin. 2013, 34, 644-650. [CrossRef] [PubMed]

10. Hung, A. Damage-Based Theories of Aging and Future Treatment Schemes. Int. J. Sci. Eng. Res. 2011, 2, 1-4.

11. Gems, D. An integrated theory of ageing in the nematode Caenorhabditis elegans. J. Anat. 2000, 197, 521-528. [CrossRef] [PubMed]

12. Back, P.; Braeckman, B.P.; Matthijssens, F. ROS in aging Caenorhabditis elegans: Damage or signaling? Oxid. Med. Cell. Longev. 2012, 2012, 608478. [CrossRef] [PubMed]

13. Lee, E.B.; Kim, J.H.; Cha, Y.S.; Kim, M.; Song, S.B.; Cha, D.S.; Jeon, H.; Eun, J.S.; Han, S.; Kim, D.K. Lifespan extending and stress resistant properties of vitexin from Vigna angularis in Caenorhabditis elegans. Biomol. Ther. 2015, 23, 582-589. [CrossRef] [PubMed]

14. Collins, J.J.; Evason, K.; Kornfeld, K. Pharmacology of delayed aging and extended lifespan of Caenorhabditis elegans. Exp. Gerontol. 2006, 41, 1032-1039. [CrossRef] [PubMed]

15. Davies, S.K.; Bundy, J.G.; Leroi, A.M. Metabolic youth in middle age: Predicting aging in Caenorhabditis elegans using metabolomics. J. Proteome Res. 2015, 14, 4603-4609. [CrossRef] [PubMed]

16. Jones, L.M.; Staffa, K.; Perally, S.; LaCourse, E.J.; Brophy, P.M.; Hamilton, J.V. Proteomic analyses of Caenorhabditis elegans dauer larvae and long-lived daf-2 mutants implicates a shared detoxification system in longevity assurance. J. Proteome Res. 2010, 9 , 2871-2881. [CrossRef] [PubMed] 
17. Saul, N.; Pietsch, K.; Stürzenbaum, S.R.; Menzel, R.; Steinberg, C.E.W. Diversity of polyphenol action in Caenorhabditis elegans: Between toxicity and longevity. J. Nat. Prod. 2011, 74, 1713-1720. [CrossRef] [PubMed]

18. Taira, N.; Nguyen, B.C.Q.; Be Tu, P.T.; Tawata, S. Effect of Okinawa Propolis on PAK1 Activity, Caenorhabditis elegans Longevity, Melanogenesis, and Growth of Cancer Cells. J. Agric. Food Chem. 2016, 64, 5484-5489. [CrossRef]

19. Chaudhary, M.K.; Rizvi, S.I. Invertebrate and vertebrate models in aging research. Biomed. Pap. Med. Fac. Univ. Palacky. Olomouc. Czech. Repub. 2019, 163, 114-121. [CrossRef]

20. Miller, D.L. There Are Worms in My Aging Research. J. Gerontol. Biol. Sci. 2019, 74, 1170-1172. [CrossRef] [PubMed]

21. Mitchell, S.J.; Scheibye-Knudsen, M.; Longo, D.L.; De Cabo, R. Animal models of aging research: Implications for human aging and age-related diseases. Annu. Rev. Anim. Biosci. 2015, 3, 283-303. [CrossRef] [PubMed]

22. Pandey, T.; Sammi, S.R.; Nooreen, Z.; Mishra, A.; Ahmad, A.; Bhatta, R.S.; Pandey, R. Anti-ageing and anti-Parkinsonian effects of natural flavonol, tambulin from Zanthoxyllum aramatum promotes longevity in Caenorhabditis elegans. Exp. Gerontol. 2019, 120, 50-61. [CrossRef]

23. Pandey, S.; Phulara, S.C.; Jha, A.; Chauhan, P.S.; Gupta, P.; Shukla, V. 3-Methyl-3-buten-1-ol (isoprenol) confers longevity and stress tolerance in Caenorhabditis elegans. Int. J. Food Sci. Nutr. 2019, 70, 595-602. [CrossRef] [PubMed]

24. Tatar, M.; Kopelman, A.; Epstein, D.; Tu, M.P.; Yin, C.M.; Garofalo, R.S. A mutant Drosophila insulin receptor homolog that extends life-span and impairs neuroendocrine function. Science 2001, 292, 107-110. [CrossRef] [PubMed]

25. Altintas, O.; Park, S.; Lee, S.J.V. The role of insulin/IGF-1 signaling in the longevity of model invertebrates, C. elegans and D. melanogaster. BMB Rep. 2016, 49, 81-92. [CrossRef] [PubMed]

26. Lan, J.; Rollins, J.A.; Zang, X.; Wu, D.; Zou, L.; Wang, Z.; Ye, C.; Wu, Z.; Kapahi, P.; Rogers, A.N.; et al. Translational Regulation of Non-autonomous Mitochondrial Stress Response Promotes Longevity. Cell Rep. 2019, 28, 1050-1062.e6. [CrossRef] [PubMed]

27. Le Bras, A. Protein aggregation contributes to aging in C. elegans. Lab Anim. 2019, 48, 200. [CrossRef]

28. Pigazzini, M.L.; Gallrein, C.; Iburg, M.; Kaminski Schierle, G.; Kirstein, J. Characterization of Amyloid Structures in Aging C. elegans Using Fluorescence Lifetime Imaging. J. Vis. Exp. 2020, 157, e61004. [CrossRef]

29. Chai, Y.; Li, W.; Feng, G.; Yang, Y.; Wang, X.; Ou, G. Live imaging of cellular dynamics during Caenorhabditis elegans postembryonic development. Nat. Protoc. 2012, 7, 2090-2102. [CrossRef]

30. Uno, M.; Nishida, E. Lifespan-regulating genes in C. elegans. Npj Aging Mech. Dis. 2016, 2, 16010. [CrossRef]

31. Olsen, A.; Vantipalli, M.C.; Lithgow, G.J. Using Caenorhabditis elegans as a model for aging and age-related diseases. Ann. N. Y. Acad. Sci. 2006, 1067, 120-128. [CrossRef] [PubMed]

32. Kenyon, C.J. The genetics of ageing. Nature 2010, 464, 504-512. [CrossRef]

33. KLASS, M.R. Culture methods Labeling. Mech. Ageing Dev. 1983, 22, 279-286. [CrossRef]

34. Schaffitzel, E.; Hertweck, M. Recent aging research in Caenorhabditis elegans. Exp. Gerontol. 2006, 41, 557-563. [CrossRef]

35. Tissenbaum, H.A. Using C. elegans for aging research. Invertebr. Reprod. Dev. 2015, 59, 59-63. [CrossRef] [PubMed]

36. Murphy, C.T.; McCarroll, S.A.; Bargmann, C.I.; Fraser, A.; Kamath, R.S.; Ahringer, J.; Li, H.; Kenyon, C. Genes that act downstream of DAF-16 to influence the lifespan of Caenorhabditis elegans. Nature 2003, 424, 277-284. [CrossRef]

37. Johnson, T.E. Subfield History: Caenorhabditis elegans as a System for Analysis of the Genetics of Aging. Sci. Aging Knowl. Environ. 2002, 2002, re4. [CrossRef] [PubMed]

38. Johnson, T.E. Advantages and disadvantages of Caenorhabditis elegans for aging research. Exp. Gerontol. 2003, 38, 1329-1332. [CrossRef] [PubMed]

39. Ahn, D.; Lee, E.B.; Kim, B.J.; Lee, S.Y.; Lee, T.G.; Ahn, M.S.; Lim, H.W.; Cha, D.S.; Jeon, H.; Kim, D.K. Antioxidant and lifespan extending property of quercetin-3-O-dirhamnoside from Curcuma longa L. in Caenorhabditis elegans. J. Korean Soc. Appl. Biol. Chem. 2014, 57, 709-714. [CrossRef]

40. Ding, A.J.; Zheng, S.Q.; Huang, X.B.; Xing, T.K.; Wu, G.S.; Sun, H.Y.; Qi, S.H.; Luo, H.R. Current Perspective in the Discovery of Anti-aging Agents from Natural Products. Nat. Products Bioprospect. 2017, 7, 335-404. [CrossRef] [PubMed]

41. Broughton, S.J.; Piper, M.D.W.; Ikeya, T.; Bass, T.M.; Jacobson, J.; Driege, Y.; Martinez, P.; Hafen, E.; Withers, D.J.; Leevers, S.J.; et al. Longer lifespan, altered metabolism, and stress resistance in Drosophila from ablation of cells making insulin-like ligands. Proc. Natl. Acad. Sci. USA 2005, 102, 3105-3110. [CrossRef] [PubMed]

42. Powers, R.W.; Kaeberlein, M.; Caldwell, S.D.; Kennedy, B.K.; Fields, S. Extension of chronological life span in yeast by decreased TOR pathway signaling. Genes Dev. 2006, 20, 174-184. [CrossRef]

43. Hsu, A.L.; Murphy, C.T.; Kenyon, C. Regulation of aging and age-related disease by DAF-16 and heat-shock factor. Science 2003, 300, 1142-1145. [CrossRef] [PubMed]

44. Mukhopadhyay, A.; Oh, S.W.; Tissenbaum, H.A. Worming pathways to and from DAF-16/FOXO. Exp. Gerontol. 2006, 41, 928-934. [CrossRef] [PubMed]

45. Kwon, E.S.; Narasimhan, S.D.; Yen, K.; Tissenbaum, H.A. A new DAF-16 isoform regulates longevity. Nature 2010, 466, 498-502. [CrossRef]

46. Wolff, S.; Ma, H.; Burch, D.; Maciel, G.A.; Hunter, T.; Dillin, A. SMK-1, an essential regulator of DAF-16-mediated longevity. Cell 2006, 124, 1039-1053. [CrossRef]

47. Kamath, R.S.; Martinez-Campos, M.; Zipperlen, P.; Fraser, A.G.; Ahringer, J. Effectiveness of specific RNA-mediated interference through ingested double-stranded RNA in Caenorhabditis elegans. Genome Biol. 2001, 2, 1-10. [CrossRef] 
48. Shukitt-Hale, B.; Lau, F.C.; Josep, J.A. Berry fruit supplementation and the aging brain. J. Agric. Food Chem. 2008, 56, 636-641. [CrossRef] [PubMed]

49. Kaunda, J.S.; Zhang, Y.J. The Genus Solanum: An Ethnopharmacological, Phytochemical and Biological Properties Review; Springer: Singapore, 2019; Volume 9, ISBN 0123456789.

50. Dehghan, E.; Zhang, Y.; Saremi, B.; Yadavali, S.; Hakimi, A.; Dehghani, M.; Goodarzi, M.; Tu, X.; Robertson, S.; Lin, R.; et al. Hydralazine induces stress resistance and extends C. elegans lifespan by activating the NRF2/SKN-1 signalling pathway. Nat. Commun. 2017, 8, 2223. [CrossRef] [PubMed]

51. Dehghan, E.; Goodarzi, M.; Saremi, B.; Lin, R.; Mirzaei, H. Hydralazine targets cAMP-dependent protein kinase leading to sirtuin1/5 activation and lifespan extension in C. elegans. Nat. Commun. 2019, 10, 4905. [CrossRef] [PubMed]

52. Gürbüz, N.; Uluişik, S.; Frary, A.; Frary, A.; Doğanlar, S. Health benefits and bioactive compounds of eggplant. Food Chem. 2018, 268, 602-610. [CrossRef] [PubMed]

53. Chen, Y.; Onken, B.; Chen, H.; Xiao, S.; Liu, X.; Driscoll, M.; Cao, Y.; Huang, Q. Mechanism of longevity extension of Caenorhabditis elegans induced by pentagalloyl glucose isolated from eucalyptus leaves. J. Agric. Food Chem. 2014, 62, 3422-3431. [CrossRef] [PubMed]

54. Ye, X.; Linton, J.M.; Schork, N.J.; Buck, L.B.; Petrascheck, M. A pharmacological network for lifespan extension in Caenorhabditis elegans. Aging Cell 2014, 13, 206-215. [CrossRef] [PubMed]

55. Altemimi, A.; Lakhssassi, N.; Baharlouei, A.; Watson, D.G.; Lightfoot, D.A. Phytochemicals: Extraction, isolation, and identification of bioactive compounds from plant extracts. Plants 2017, 6, 42. [CrossRef] [PubMed]

56. Jenzer Bern, H.; Sadeghi Bern, L. Phytochemicals: Sources and biological functions. J. Pharmacogn. Phytochem. JPP 2016, 339, 339-341.

57. Banu, K.S.; Cathrine, L. General Techniques Involved in Phytochemical Analysis. Int. J. Adv. Res. Chem. Sci. 2015, 2, 25-32.

58. Tiwari, P.; Kumar, B.; Mandeep, K.; Kaur, G.; Kaur, H. Phytochemical screening and Extraction: A Review. Int. Pharm. Sci. 2011, 1, 98-106.

59. Papaevgeniou, N.; Sakellari, M.; Jha, S.; Tavernarakis, N.; Holmberg, C.I.; Gonos, E.S.; Chondrogianni, N. 18 $\alpha$-Glycyrrhetinic acid proteasome activator decelerates aging and Alzheimer's disease progression in Caenorhabditis elegans and neuronal cultures. Antioxid. Redox Signal. 2016, 25, 855-869. [CrossRef] [PubMed]

60. Papaevgeniou, N.; Chondrogianni, N. Anti-aging and Anti-aggregation Properties of Polyphenolic Compounds in C. elegans. Curr. Pharm. Des. 2018, 24, 2107-2120. [CrossRef] [PubMed]

61. Abdel-Moneim, A.M.E.; Shehata, A.M.; Alzahrani, S.O.; Shafi, M.E.; Mesalam, N.M.; Taha, A.E.; Swelum, A.A.; Arif, M.; Fayyaz, M.; Abd El-Hack, M.E. The role of polyphenols in poultry nutrition. J. Anim. Physiol. Anim. Nutr. 2020, 104, 1851-1866. [CrossRef] [PubMed]

62. Banjarnahor, S.D.S.; Artanti, N. Antioxidant properties of flavonoids. Med. J. Indones. 2014, 23, 239-244. [CrossRef]

63. Lin, C.; Xiao, J.; Xi, Y.; Zhang, X.; Zhong, Q.; Zheng, H.; Cao, Y.; Chen, Y. Rosmarinic acid improved antioxidant properties and healthspan via the IIS and MAPK pathways in Caenorhabditis elegans. BioFactors 2019, 45, 774-787. [CrossRef] [PubMed]

64. Liao, V.H.C.; Yu, C.W.; Chu, Y.J.; Li, W.H.; Hsieh, Y.C.; Wang, T.T. Curcumin-mediated lifespan extension in Caenorhabditis elegans. Mech. Ageing Dev. 2011, 132, 480-487. [CrossRef] [PubMed]

65. Zheng, S.Q.; Huang, X.B.; Xing, T.K.; Ding, A.J.; Wu, G.S.; Luo, H.R. Chlorogenic acid extends the lifespan of Caenorhabditis elegans via Insulin/IGF-1 signaling pathway. J. Gerontol. Ser. A Biol. Sci. Med. Sci. 2017, 72, 464-472. [CrossRef]

66. Abbas, S.; Wink, M. Epigallocatechin gallate inhibits beta amyloid oligomerization in Caenorhabditis elegans and affects the daf-2/insulin-like signaling pathway. Phytomedicine 2010, 17, 902-909. [CrossRef]

67. Abbas, S.; Wink, M. Epigallocatechin gallate from green tea (Camellia sinensis) increases lifespan and stress resistance in Caenorhabditis elegans. Planta Med. 2009, 75, 216-221. [CrossRef]

68. Ayuda-Durán, B.; González-Manzano, S.; Miranda-Vizuete, A.; Dueñas, M.; Santos-Buelga, C.; González-Paramás, A.M. Epicatechin modulates stress-resistance in C. elegans via insulin/IGF-1 signaling pathway. PLoS ONE 2019, 14, e0199483. [CrossRef] [PubMed]

69. Büchter, C.; Ackermann, D.; Havermann, S.; Honnen, S.; Chovolou, Y.; Fritz, G.; Kampkötter, A.; Wätjen, W. Myricetin-mediated lifespan extension in Caenorhabditis elegans is modulated by DAF-16. Int. J. Mol. Sci. 2013, 14, 11895-11914. [CrossRef] [PubMed]

70. Havermann, S.; Chovolou, Y.; Humpf, H.U.; Wätjen, W. Caffeic acid phenethylester increases stress resistance and enhances lifespan in Caenorhabditis elegans by modulation of the insulin-like DAF-16 signalling pathway. PLoS ONE 2014, 9, e100256. [CrossRef] [PubMed]

71. Asthana, J.; Yadav, D.; Pant, A.; Yadav, A.K.; Gupta, M.M.; Pandey, R. Acacetin 7-O- $\alpha$-1-rhamnopyranosyl (1-2) $\beta$-Dxylopyranoside elicits life-span extension and stress resistance in Caenorhabditis elegans. J. Gerontol. Ser. A Biol. Sci. Med. Sci. 2016, 71, 1160-1168. [CrossRef] [PubMed]

72. Sater, H.M.; Bizzio, L.N.; Tieman, D.M.; Muñoz, P.D. A Review of the Fruit Volatiles Found in Blueberry and Other Vaccinium Species. J. Agric. Food Chem. 2020, 68, 5777-5786. [CrossRef] [PubMed]

73. Bass, T.M.; Weinkove, D.; Houthoofd, K.; Gems, D.; Partridge, L. Effects of resveratrol on lifespan in Drosophila melanogaster and Caenorhabditis elegans. Mech. Ageing Dev. 2007, 128, 546-552. [CrossRef] [PubMed] 
74. Zarse, K.; Bossecker, A.; Müller-Kuhrt, L.; Siems, K.; Hernandez, M.A.; Berendsohn, W.G.; Birringer, M.; Ristow, M. The phytochemical glaucarubinone promotes mitochondrial metabolism, reduces body fat, and extends lifespan of Caenorhabditis elegans. Horm. Metab. Res. 2011, 43, 241-243. [CrossRef]

75. Morselli, E.; Maiuri, M.C.; Markaki, M.; Megalou, E.; Pasparaki, A.; Palikaras, K.; Criollo, A.; Galluzzi, L.; Malik, S.A.; Vitale, I.; et al. Caloric restriction and resveratrol promote longevity through the Sirtuin-1-dependent induction of autophagy. Cell Death Dis. 2010, 1, e10. [CrossRef] [PubMed]

76. Kampkötter, A.; Timpel, C.; Zurawski, R.F.; Ruhl, S.; Chovolou, Y.; Proksch, P.; Wätjen, W. Increase of stress resistance and lifespan of Caenorhabditis elegans by quercetin. Comp. Biochem. Physiol. B Biochem. Mol. Biol. 2008, 149, 314-323. [CrossRef]

77. Grünz, G.; Haas, K.; Soukup, S.; Klingenspor, M.; Kulling, S.E.; Daniel, H.; Spanier, B. Structural features and bioavailability of four flavonoids and their implications for lifespan-extending and antioxidant actions in C. elegans. Mech. Ageing Dev. 2012, 133, 1-10. [CrossRef]

78. Saul, N.; Pietsch, K.; Menzel, R.; Stürzenbaum, S.R.; Steinberg, C.E.W. Catechin induced longevity in C. elegans: From key regulator genes to disposable soma. Mech. Ageing Dev. 2009, 130, 477-486. [CrossRef]

79. Havermann, S.; Rohrig, R.; Chovolou, Y.; Humpf, H. Molecular Effects of Baicalein in Hct116 Cells and Caenorhabditis elegans: Activation of the Nrf2 Signaling Pathway and Prolongation of Lifespan. J. Agric. Food Chem. 2013, 61, 2158-2164. [CrossRef]

80. Kampkötter, A.; Gombitang Nkwonkam, C.; Zurawski, R.F.; Timpel, C.; Chovolou, Y.; Wätjen, W.; Kahl, R. Effects of the flavonoids kaempferol and fisetin on thermotolerance, oxidative stress and FoxO transcription factor DAF-16 in the model organism Caenorhabditis elegans. Arch. Toxicol. 2007, 81, 849-858. [CrossRef]

81. Bartholome, A.; Kampkötter, A.; Tanner, S.; Sies, H.; Klotz, L.O. Epigallocatechin gallate-induced modulation of FoxO signaling in mammalian cells and C. elegans: FoxO stimulation is masked via PI3K/Akt activation by hydrogen peroxide formed in cell culture. Arch. Biochem. Biophys. 2010, 501, 58-64. [CrossRef]

82. Pietsch, K.; Saul, N.; Menzel, R.; Stürzenbaum, S.R.; Steinberg, C.E.W. Quercetin mediated lifespan extension in Caenorhabditis elegans is modulated by age-1, daf-2, sek-1 and unc-43. Biogerontology 2009, 10, 565-578. [CrossRef]

83. Surco-Laos, F.; Dueñas, M.; González-Manzano, S.; Cabello, J.; Santos-Buelga, C.; González-Paramás, A.M. Influence of catechins and their methylated metabolites on lifespan and resistance to oxidative and thermal stress of Caenorhabditis elegans and epicatechin uptake. Food Res. Int. 2012, 46, 514-521. [CrossRef]

84. Lublin, A.; Isoda, F.; Patel, H.; Yen, K.; Nguyen, L.; Hajje, D.; Schwartz, M.; Mobbs, C. FDA-approved drugs that protect mammalian neurons from glucose toxicity slow aging dependent on $\mathrm{Cbp}$ and protect against proteotoxicity. PLoS ONE 2011, 6, e27762. [CrossRef] [PubMed]

85. Pietsch, K.; Saul, N.; Chakrabarti, S.; Stürzenbaum, S.R.; Menzel, R.; Steinberg, C.E.W. Hormetins, antioxidants and prooxidants: Defining quercetin-, caffeic acid- and rosmarinic acid-mediated life extension in C. elegans. Biogerontology 2011, 12, 329-347. [CrossRef] [PubMed]

86. Asthana, J.; Mishra, B.N.; Pandey, R. Acacetin promotes healthy aging by altering stress response in Caenorhabditis elegans. Free Radic. Res. 2016, 50, 861-874. [CrossRef] [PubMed]

87. Asthana, J.; Yadav, A.K.; Pant, A.; Pandey, S.; Gupta, M.M.; Pandey, R. Specioside ameliorates oxidative stress and promotes longevity in Caenorhabditis elegans. Comp. Biochem. Physiol. Part C Toxicol. Pharmacol. 2015, 169, 25-34. [CrossRef] [PubMed]

88. Dueñas, M.; Surco-Laos, F.; González-Manzano, S.; González-Paramás, A.M.; Gómez-Orte, E.; Cabello, J.; Santos-Buelga, C. Deglycosylation is a key step in biotransformation and lifespan effects of quercetin-3-O-glucoside in Caenorhabditis elegans. Pharmacol. Res. 2013, 76, 41-48. [CrossRef]

89. Surco-Laos, F.; Cabello, J.; Gómez-Orte, E.; González-Manzano, S.; González-Paramás, A.M.; Santos-Buelga, C.; Dueñas, M. Effects of O-methylated metabolites of quercetin on oxidative stress, thermotolerance, lifespan and bioavailability on Caenorhabditis elegans. Food Funct. 2011, 2, 445-456. [CrossRef] [PubMed]

90. Cai, W.J.; Huang, J.H.; Zhang, S.Q.; Wu, B.; Kapahi, P.; Zhang, X.M.; Shen, Z.Y. Icariin and its derivative icariside II extend healthspan via insulin/IGF-1 pathway in C. elegans. PLoS ONE 2011, 6, e28835. [CrossRef]

91. Büchter, C.; Havermann, S.; Koch, K.; Wätjen, W. Isoxanthohumol, a constituent of hop (Humulus lupulus L.), increases stress resistance in Caenorhabditis elegans dependent on the transcription factor DAF-16. Eur. J. Nutr. 2016, 55, 257-265. [CrossRef] [PubMed]

92. Lee, E.B.; Ahn, D.; Kim, B.J.; Lee, S.Y.; Seo, H.W.; Cha, Y.S.; Jeon, H.; Eun, J.S.; Cha, D.S.; Kim, D.K. Genistein from vigna angularis extends lifespan in Caenorhabditis elegans. Biomol. Ther. 2015, 23, 77-83. [CrossRef] [PubMed]

93. Benedetti, M.G.; Foster, A.L.; Vantipalli, M.C.; White, M.P.; Sampayo, J.N.; Gill, M.S.; Olsen, A.; Lithgow, G.J. Compounds that confer thermal stress resistance and extended lifespan. Exp. Gerontol. 2008, 43, 882-891. [CrossRef] [PubMed]

94. Schlernitzauer, A.; Oiry, C.; Hamad, R.; Galas, S.; Cortade, F.; Chabi, B.; Casas, F.; Pessemesse, L.; Fouret, G.; Feillet-Coudray, C.; et al. Chicoric acid is an antioxidant molecule that stimulates AMP kinase pathway in L6 myotubes and extends lifespan in Caenorhabditis elegans. PLoS ONE 2013, 8, e78788. [CrossRef]

95. Saul, N.; Pietsch, K.; Menzel, R.; Stürzenbaum, S.R.; Steinberg, C.E.W. The longevity effect of tannic acid in Caenorhabditis elegans: Disposable soma meets hormesis. J. Gerontol. Ser. A Biol. Sci. Med. Sci. 2010, 65 A, 626-635. [CrossRef]

96. Lee, J.; Kwon, G.; Park, J.; Kim, J.K.; Lim, Y.H. Brief Communication: SIR-2.1-dependent lifespan extension of Caenorhabditis elegans by oxyresveratrol and resveratrol. Exp. Biol. Med. 2016, 241, 1757-1763. [CrossRef] [PubMed] 
97. Büchter, C.; Zhao, L.; Havermann, S.; Honnen, S.; Fritz, G.; Proksch, P.; Wätjen, W. TSG (2,3,5,4'-Tetrahydroxystilbene-2-O- $\beta$-Dglucoside) from the Chinese herb Polygonum multiflorum increases lifespan and stress resistance of Caenorhabditis elegans. Oxid. Med. Cell. Longev. 2015, 2015, 124357. [CrossRef]

98. Wen, H.; Gao, X.; Qin, J. Probing the anti-aging role of polydatin in Caenorhabditis elegans on a chip. Integr. Biol. (UK) 2014, 6, 35-43. [CrossRef]

99. Shen, P.; Yue, Y.; Sun, Q.; Kasireddy, N.; Kim, K.H.; Park, Y. Piceatannol extends the lifespan of Caenorhabditis elegans via DAF-16. BioFactors 2017, 43, 379-387. [CrossRef]

100. Ryu, D.; Mouchiroud, L.; Andreux, P.A.; Katsyuba, E.; Moullan, N.; Nicolet-Dit-Félix, A.A.; Williams, E.G.; Jha, P.; Lo Sasso, G.; Huzard, D.; et al. Urolithin A induces mitophagy and prolongs lifespan in C. elegans and increases muscle function in rodents. Nat. Med. 2016, 22, 879-888. [CrossRef] [PubMed]

101. Yaguchi, Y.; Komura, T.; Kashima, N.; Tamura, M.; Kage-Nakadai, E.; Saeki, S.; Terao, K.; Nishikawa, Y. Influence of oral supplementation with sesamin on longevity of Caenorhabditis elegans and the host defense. Eur. J. Nutr. 2014, 53, 1659-1668. [CrossRef] [PubMed]

102. Su, S.; Wink, M. Natural lignans from Arctium lappa as antiaging agents in Caenorhabditis elegans. Phytochemistry 2015, 117, 340-350. [CrossRef]

103. Cañuelo, A.; Gilbert-López, B.; Pacheco-Liñán, P.; Martínez-Lara, E.; Siles, E.; Miranda-Vizuete, A. Tyrosol, a main phenol present in extra virgin olive oil, increases lifespan and stress resistance in Caenorhabditis elegans. Mech. Ageing Dev. 2012, 133, 563-574. [CrossRef]

104. Ayyadevara, S.; Bharill, P.; Dandapat, A.; Hu, C.; Khaidakov, M.; Mitra, S.; Shmookler Reis, R.J.; Mehta, J.L. Aspirin inhibits oxidant stress, reduces age-associated functional declines, and extends lifespan of Caenorhabditis elegans. Antioxid. Redox Sign. 2013, 18, 481-490. [CrossRef]

105. Nguyen, T.T.; Caito, S.W.; Zackert, W.E.; West, J.D.; Zhu, S.; Aschner, M.; Fessel, J.P.; Roberts, L.J. Scavengers of reactive $\gamma$-ketoaldehydes extend Caenorhabditis elegans lifespan and healthspan through protein-level interactions with SIR-2.1 and ETS-7. Aging (Albany NY) 2016, 8, 1759-1780. [CrossRef]

106. Heidler, T.; Hartwig, K.; Daniel, H.; Wenzel, U. Caenorhabditis elegans lifespan extension caused by treatment with an orally active ROS-generator is dependent on DAF-16 and SIR-2.1. Biogerontology 2010, 11, 183-195. [CrossRef]

107. Li, H.; Yu, X.; Meng, F.; Zhao, Z.; Guan, S.; Wang, L. Ferulic acid supplementation increases lifespan and stress resistance via insulin/IGF-1 signaling pathway in C. elegans. Int. J. Mol. Sci. 2021, 22, 4279. [CrossRef] [PubMed]

108. Wang, G.; Tang, W.; Bidigare, R.R. Terpenoids as therapeutic drugs and pharmaceutical agents. In Natural Products: Drug Discovery and Therapeutic Medicine; Humana Press: Totowa, NJ, USA, 2005; pp. 197-227. ISBN 9781588293831.

109. Lin, C.; Zhang, X.; Xiao, J.; Zhong, Q.; Kuang, Y.; Cao, Y.; Chen, Y. Effects on longevity extension and mechanism of action of carnosic acid in: Caenorhabditis elegans. Food Funct. 2019, 10, 1398-1410. [CrossRef] [PubMed]

110. Lin, C.; Zhang, X.; Su, Z.; Xiao, J.; Lv, M.; Cao, Y.; Chen, Y. Carnosol improved lifespan and healthspan by promoting antioxidant capacity in Caenorhabditis elegans. Oxid. Med. Cell. Longev. 2019, 2019, 5958043. [CrossRef] [PubMed]

111. Pant, A.; Saikia, S.K.; Shukla, V.; Asthana, J.; Akhoon, B.A.; Pandey, R. Beta-caryophyllene modulates expression of stress response genes and mediates longevity in Caenorhabditis elegans. Exp. Gerontol. 2014, 57, 81-95. [CrossRef] [PubMed]

112. Shukla, V.; Yadav, D.; Phulara, S.C.; Gupta, M.M.; Saikia, S.K.; Pandey, R. Longevity-promoting effects of 4-hydroxy-E-globularinin in Caenorhabditis elegans. Free Radic. Biol. Med. 2012, 53, 1848-1856. [CrossRef]

113. Zhang, J.; Lu, L.; Zhou, L. Oleanolic acid activates daf-16 to increase lifespan in Caenorhabditis elegans. Biochem. Biophys. Res. Commun. 2015, 468, 843-849. [CrossRef] [PubMed]

114. Li, J.; Chotiko, A.; Chouljenko, A.; Gao, C.; Zheng, J.; Sathivel, S. Delivery of alpha-tocopherol through soluble dietary fibre-based nanofibres for improving the life span of Caenorhabditis elegans. Int. J. Food Sci. Nutr. 2019, 70, 172-181. [CrossRef]

115. Akhoon, B.A.; Pandey, S.; Tiwari, S.; Pandey, R. Withanolide A Offers Neuroprotection, Ameliorates Stress Resistance and Prolongs the Life Expectancy of Caenorhabditis Elegans; Elsevier B.V.: Amsterdam, The Netherlands, 2016; Volume 78, ISBN 5222718530.

116. Pant, A.; Asthana, J.; Yadav, A.K.; Rathor, L.; Srivastava, S.; Gupta, M.M.; Pandey, R. Verminoside mediates life span extension and alleviates stress in Caenorhabditis elegans. Free Radic. Res. 2015, 49, 1384-1392. [CrossRef] [PubMed]

117. Negi, H.; Shukla, A.; Khan, F.; Pandey, R. 3ß-Hydroxy-urs-12-en-28-oic acid prolongs lifespan in C. elegans by modulating JNK-1. Biochem. Biophys. Res. Commun. 2016, 480, 539-543. [CrossRef]

118. Shukla, V.; Phulara, S.C.; Yadav, D.; Tiwari, S.; Kaur, S.; Gupta, M.M.; Nazir, A.; Pandey, R. Iridoid Compound 10-O-transp-Coumaroylcatalpol Extends Longevity and Reduces Alpha Synuclein Aggregation in Caenorhabditis elegans. CNS Neurol. Disord.-Drug Targets 2013, 11, 984-992. [CrossRef] [PubMed]

119. Lashmanova, E.; Proshkina, E.; Zhikrivetskaya, S.; Shevchenko, O.; Marusich, E.; Leonov, S.; Melerzanov, A.; Zhavoronkov, A.; Moskalev, A. Fucoxanthin increases lifespan of Drosophila melanogaster and Caenorhabditis elegans. Pharmacol. Res. 2015, 100, 228-241. [CrossRef] [PubMed]

120. Seo, H.W.; Cheon, S.M.; Lee, M.H.; Kim, H.J.; Jeon, H.; Cha, D.S. Catalpol modulates lifespan via DAF-16/FOXO and SKN-1/Nrf2 activation in Caenorhabditis elegans. Evidence-Based Complement. Altern. Med. 2015, 2015, 524878. [CrossRef] [PubMed]

121. Sayed, A.A.R. Ferulsinaic acid attenuation of advanced glycation end products extends the lifespan of Caenorhabditis elegans. J. Pharm. Pharmacol. 2011, 63, 423-428. [CrossRef] [PubMed] 
122. Kim, J.; Kang, Y.-G.; Lee, J.; Choi, D.; Cho, Y.; Shin, J.-M.; Park, J.S.; Lee, J.H.; Kim, W.G.; Seo, D.B.; et al. The natural phytochemical dehydroabietic acid is an anti-aging reagent that mediates the direct activation of SIRT1. Mol. Cell. Endocrinol. 2015, 412, 216-225. [CrossRef] [PubMed]

123. Lu, M.; Tan, L.; Zhou, X.G.; Yang, Z.L.; Zhu, Q.; Chen, J.N.; Luo, H.R.; Wu, G.S. Secoisolariciresinol Diglucoside Delays the Progression of Aging-Related Diseases and Extends the Lifespan of Caenorhabditis elegans via DAF-16 and HSF-1. Oxid. Med. Cell. Longev. 2020, 2020, 1293935. [CrossRef] [PubMed]

124. Roy, A. A review on the alkaloids an important therapeutic compound from plants. Int. J. Plant Biotechnol. 2017, 3, 1-9. [CrossRef]

125. Hardie, D.G. AMP-activated protein kinase-an energy sensor that regulates all aspects of cell function. Genes Dev. 2011, 25, 1895-1908. [CrossRef]

126. Srivastava, D.; Arya, U.; SoundaraRajan, T.; Dwivedi, H.; Kumar, S.; Subramaniam, J.R. Reserpine can confer stress tolerance and lifespan extension in the nematode C. elegans. Biogerontology 2008, 9, 309-316. [CrossRef] [PubMed]

127. Li, H.; Roxo, M.; Cheng, X.; Zhang, S.; Cheng, H.; Wink, M. Pro-oxidant and lifespan extension effects of caffeine and related methylxanthines in Caenorhabditis elegans. Food Chem. X 2019, 1, 100005. [CrossRef] [PubMed]

128. Bridi, J.C.; de Almeida Barros, A.G.; Sampaio, L.R.; Damásio Ferreira, J.C.; Antunes Soares, F.A.; Romano-Silva, M.A. Lifespan extension induced by caffeine in Caenorhabditis elegans is partially dependent on adenosine signaling. Front. Aging Neurosci. 2015, 7, 220. [CrossRef] [PubMed]

129. Fang, E.F.; Waltz, T.B.; Kassahun, H.; Lu, Q.; Kerr, J.S.; Morevati, M.; Fivenson, E.M.; Wollman, B.N.; Marosi, K.; Wilson, M.A.; et al. Tomatidine enhances lifespan and healthspan in C. elegans through mitophagy induction via the SKN-1/Nrf2 pathway. Sci. Rep. 2017, 7, 46208. [CrossRef] [PubMed]

130. Morselli, E.; Mariño, G.; Bennetzen, M.V.; Eisenberg, T.; Megalou, E.; Schroeder, S.; Cabrera, S.; Bénit, P.; Rustin, P.; Criollo, A.; et al. Spermidine and resveratrol induce autophagy by distinct pathways converging on the acetylproteome. J. Cell Biol. 2011, 192, 615-629. [CrossRef] [PubMed]

131. Eisenberg, T.; Knauer, H.; Schauer, A.; Büttner, S.; Ruckenstuhl, C.; Carmona-Gutierrez, D.; Ring, J.; Schroeder, S.; Magnes, C.; Antonacci, L.; et al. Induction of autophagy by spermidine promotes longevity. Nat. Cell Biol. 2009, 11, 1305-1314. [CrossRef] [PubMed]

132. Wang, E.; Wink, M. Chlorophyll enhances oxidative stress tolerance in Caenorhabditis elegans and extends its lifespan. PeerJ 2016, 4, e1879. [CrossRef]

133. Wu, J.Z.; Huang, J.H.; Khanabdali, R.; Kalionis, B.; Xia, S.J.; Cai, W.J. Pyrroloquinoline quinone enhances the resistance to oxidative stress and extends lifespan upon DAF-16 and SKN-1 activities in C. elegans. Exp. Gerontol. 2016, 80, 43-50. [CrossRef]

134. Lu, L.; Zhao, X.; Zhang, J.; Li, M.; Qi, Y.; Zhou, L. Calycosin promotes lifespan in Caenorhabditis elegans through insulin signaling pathway via daf-16, age-1 and daf-2. J. Biosci. Bioeng. 2017, 124, 1-7. [CrossRef] [PubMed]

135. Peixoto, H.; Roxo, M.; Silva, E.; Valente, K.; Braun, M.; Wang, X.; Wink, M. Bark extract of the amazonian tree endopleura uchi (humiriaceae) extends lifespan and enhances stress resistance in Caenorhabditis elegans. Molecules 2019, 24, 915. [CrossRef] [PubMed]

136. Peixoto, H.; Roxo, M.; Koolen, H.; Da Silva, F.; Silva, E.; Braun, M.S.; Wang, X.; Wink, M. Calycophyllum spruceanum (Benth.), the amazonian "tree of youth" prolongs longevity and enhances stress resistance in Caenorhabditis elegans. Molecules 2018, 23, 534. [CrossRef] [PubMed]

137. Rangsinth, P.; Prasansuklab, A.; Duangjan, C.; Gu, X.; Meemon, K.; Wink, M.; Tencomnao, T. Leaf extract of Caesalpinia mimosoides enhances oxidative stress resistance and prolongs lifespan in Caenorhabditis elegans. BMC Complement. Altern. Med. 2019, 19, 164. [CrossRef] [PubMed]

138. Tambara, A.L.; de Los Santos Moraes, L.; Dal Forno, A.H.; Boldori, J.R.; Gonçalves Soares, A.T.; de Freitas Rodrigues, C.; Mariutti, L.R.B.; Mercadante, A.Z.; de Ávila, D.S.; Denardin, C.C. Purple pitanga fruit (Eugenia uniflora L.) protects against oxidative stress and increase the lifespan in Caenorhabditis elegans via the DAF-16/FOXO pathway. Food Chem. Toxicol. 2018, 120, 639-650. [CrossRef] [PubMed]

139. Duangjan, C.; Rangsinth, P.; Gu, X.; Wink, M.; Tencomnao, T. Lifespan extending and oxidative stress resistance properties of a leaf extracts from anacardium occidentale L. in Caenorhabditis elegans. Oxid. Med. Cell. Longev. 2019, 2019, 9012396. [CrossRef]

140. Duangjan, C.; Rangsinth, P.; Gu, X.; Zhang, S.; Wink, M.; Tencomnao, T. Glochidion zeylanicum leaf extracts exhibit lifespan extending and oxidative stress resistance properties in Caenorhabditis elegans via DAF-16/FoxO and SKN-1/Nrf-2 signaling pathways. Phytomedicine 2019, 64, 153061. [CrossRef] [PubMed]

141. Koch, K.; Weldle, N.; Baier, S.; Büchter, C.; Wätjen, W. Hibiscus sabdariffa L. extract prolongs lifespan and protects against amyloid- $\beta$ toxicity in Caenorhabditis elegans: Involvement of the FoxO and Nrf2 orthologues DAF-16 and SKN-1. Eur. J. Nutr. 2020, 59, 137-150. [CrossRef] [PubMed]

142. Rathor, L.; Pant, A.; Awasthi, H.; Mani, D.; Pandey, R. An antidiabetic polyherbal phytomedicine confers stress resistance and extends lifespan in Caenorhabditis elegans. Biogerontology 2017, 18, 131-147. [CrossRef] [PubMed]

143. Pandey, S.; Phulara, S.C.; Mishra, S.K.; Bajpai, R.; Kumar, A.; Niranjan, A.; Lehri, A.; Upreti, D.K.; Chauhan, P.S. Betula utilis extract prolongs life expectancy, protects against amyloid- $\beta$ toxicity and reduces Alpha Synuclien in Caenorhabditis elegans via DAF-16 and SKN-1. Comp. Biochem. Physiol. Part-C Toxicol. Pharmacol. 2020, 228, 108647. [CrossRef] [PubMed] 
144. Sayed, S.M.A.; Siems, K.; Schmitz-Linneweber, C.; Luyten, W.; Saul, N. Enhanced healthspan in Caenorhabditis elegans treated with extracts from the traditional chinese medicine plants Cuscuta chinensis Lam. and Eucommia ulmoides Oliv. Front. Pharmacol. 2021, 12, 604435. [CrossRef]

145. O'Reilly, L.P.; Luke, C.J.; Perlmutter, D.H.; Silverman, A.G.; Pak, S.C. C. elegans in high-throughput drug discovery. Adv. Drug Deliv. Rev. 2014, 23, 247-253. [CrossRef] [PubMed]

146. Ben-Yakar, A. High-content and high-throughput in vivo drug screening platforms using microfluidics. Assay Drug Dev. Technol. 2019, 17, 8-13. [CrossRef] [PubMed]

147. Banerjee, S.; Riordan, M.; Bhat, M.A. Genetic aspects of autism spectrum disorders: Insights from animal models. Front. Cell. Neurosci. 2014, 8, 58. [CrossRef] [PubMed]

148. Schmeisser, K.; Parker, J.A. Worms on the spectrum-C. elegans models in autism research. Exp. Neurol. 2018, $299,199-206$. [CrossRef] [PubMed] 